Pacific Journal of Mathematics

DOMAINS IN RIEMANNIAN MANIFOLDS AND INVERS 


\section{DOMAINS IN RIEMANNIAN MANIFOLDS AND INVERSE SPECTRAL GEOMETRY}

\section{HAROLd DONNElly AND JefFrey LeE}

The asymptotic formula of Weyl, $\left(\lambda_{k}\right)^{n / 2} \sim c(n) k / \operatorname{vol}(D)$, shows that the volume of a bounded domain $D$ in an $n$ dimensional Riemannian manifold is determined by the Dirichlet spectrum, $\left\{\lambda_{k}\right\}$, of the domain. Also, the asymptotic expansion for the trace of the Dirichlet heat kernel of a smooth bounded domain shows that the volume of the boundary is determined by the spectrum of the domain. However, these asymptotic expressions do not tell us, in themselves, how many eigenvalues one needs in order to approximate the volume of the domain or its boundary to within a prescribed error. We give several results which answer this question, for certain types of domains, in terms of the geometry of the ambient manifold. Some knowledge of the domain is needed. In particular, the distance from the boundary to the boundary's cut locus in the ambient manifold is relevant. Thus, we also prove a purely differential geometric structure theorem relating the distance from the boundary of the domain to the interior part of its cut locus, to the principal curvatures of the boundary.

1. Introduction. We consider the Laplace operator $\Delta$ defined on a smooth relatively compact domain $D$ in a Riemannian manifold $M^{n}$. By domain we will always mean connected open set. Let $\left\{-\lambda_{i}\right\}$ be the set of eigenvalues or spectrum of $\Delta$ with Dirichlet boundary conditions indexed in increasing order and including multiplicity:

$$
0<\lambda_{1} \leq \lambda_{2} \leq \lambda_{3} \leq \cdots \uparrow \infty .
$$

The spectrum of $D$ determines certain aspects of the geometry of $D$. For instance Weyl's formula:

$$
\lim _{k \rightarrow \infty} \frac{\lambda_{k}}{k^{2 / n}}=\frac{c_{n}}{\operatorname{vol}(D)^{2 / n}}
$$

shows that the spectrum determines the volume of $D$. More information is given by the asymptotic formula for $\operatorname{trace}\left(e^{t \Delta}\right)$, for sufficiently small $t$,

$$
\sum_{i=1}^{\infty} e^{-\lambda_{i} t}=\frac{1}{(4 \pi t)^{n / 2}}\left\{\operatorname{vol}(D)-\frac{\sqrt{\pi}}{2} \operatorname{vol}(\partial D) t^{1 / 2}+O(t)\right\} .
$$

In particular, $\operatorname{vol}(\partial D)$ is determined by the spectrum of $\Delta$. 
An important point is that these asymptotic expressions, in themselves, do not tell us how many eigenvalues one would need to estimate the given geometric quantity (e.g. volume) to within a prescribed error. This provides motivation for much of what we do here. One would like to be able to estimate geometric invariants without assuming too much a priori knowledge of the geometry of $D$. For example, it would be unfortunate if in order to tell how large to take $k$ in Weyl's formula to know the volume to within some $\varepsilon>0$, we needed to know the volume itself. Along these lines, there is the following result [T] of $\mathrm{Li}$ and Yau for convex domains in $\mathbb{R}^{n}$.

Theorem 1.1 ( $L i$ and Yau). Let $D$ be a convex domain in $\mathbb{R}^{n}$. Given $\varepsilon>0$ there exists an $N_{0}$ depending only on $\varepsilon, n, \lambda_{1}$ and $\mathscr{M}\left(\beta \lambda_{1}\right)$ such that for $k>N_{0}$

$$
\left|\frac{\lambda_{k}}{k^{2 / n}}-\frac{c_{n}}{(\operatorname{vol}(D))^{2 / n}}\right|<\varepsilon
$$

where $\beta>8 \pi^{-2} n(n+4)$ and $\mathscr{M}\left(\beta \lambda_{1}\right)=\max \left\{j: \lambda_{j} \leq \beta \lambda_{1}\right\}$.

Notice that $\mathscr{M}\left(\lambda_{1} \beta\right)$ is determined by a finite part of the spectrum. It is worth pointing out that $\mathscr{M}\left(\lambda_{1} \beta\right)$ really occurs in the proof as part of an upper bound for the out-radius:

$$
R_{\text {out }} \leq \frac{\pi \mathscr{M}\left(\lambda_{1} \beta\right)}{\sqrt{\lambda_{1}}}
$$

The derivation of this estimate uses properties of Euclidean space that fail in spaces of negative curvature. One can, however, give a result analogous to the above theorem for geodesically convex domains in hyperbolic space if one is willing to replace $\mathscr{M}\left(\lambda_{1} \beta\right)$ by some assumed crude upper bound on the out-radius [L].

We employ heat kernel remainder estimates to extend Theorem 1.1 for domains in manifolds of variable curvature. The authors of the present paper have obtained some results of this type [D-L]. In [D-L], as in [L] and Theorems 2.3 and 2.13 below, one must in general assume an a priori estimate on the out-radius as part of the data. In case the ambient manifold is compact this is not so much to ask since it is more acceptable to assume something about the ambient manifold and we can replace the out-radius bound by a bound on the diameter of the ambient manifold. The assumed out-radius bound provides an upper bound for the surface area and volume which is needed to obtain the results in $[\mathbf{D}-\mathbf{L}]$. In $[\mathbf{D}-\mathbf{L}]$ the authors give a way of estimating $\operatorname{vol} D$ 
up to arbitrary accuracy from a finite part of the spectrum of $\Delta$ on $D$. As in Theorem 1.1 above, $D$ is required to satisfy an appropriate convexity condition. Whereas the results of [D-L] deal only with the volume of the domain, in the present paper we obtain analogous results for the volume of the boundary. In particular, Theorem 2.13 below shows that one can estimate vol $\partial D$ up to arbitrary accuracy from a finite part of the Dirichlet spectrum of $\Delta$ on $D$. However in Theorem 2.13 we do not need convexity. This is true for most results of $\S 2$. In [D-L] we must assume some knowledge of both $D$ and the geometry of the ambient manifold $M$. The situation for Theorem 2.13 below is similar, and in particular, we need a lower bound for the distance from $\partial D$ to its cut-locus. In other words, we need to add to our data a lower bound for $c_{\partial D}$, where $c_{\partial D}>0$ is the supremum of all numbers such that the normal exponential map $\exp _{\partial D}^{\perp}: \nu(\partial D) \rightarrow M$ is a diffeomorphism on $\Gamma=\left\{\nu \in \nu(\partial D):|\nu|<c_{\partial D}\right\}$. Once a positive lower bound for $c_{\partial D}$ is allowed as data, the out-radius estimate can be eliminated. This is shown in Theorems 2.16 and 2.17 below. In fact, we will show in Theorem 2.15 that, in this case, an upper bound for the surface area and volume of $D$ can be determined from a finite part of the spectrum of $D$. In Theorem 2.14 an upper bound for the out-radius itself is given.

Finally, it is desirable to have a way of obtaining the bounds on $c_{\partial D}$ in terms of boundary curvature and the geometry of $M$. This is also motivated by the fact that it is boundary curvature that plays a role in the asymptotic expansion of the heat kernel for $D$. The estimation of the distance to the part of the cut locus of $\partial D$ interior to $D$ is of interest in itself and is the subject of the last half of the present paper. The following fact also provides motivation for the results of $\S 3$. Let $D$ be a convex domain in $\mathbb{R}^{n}$ with $\partial D$ having principal curvatures bounded above by $\frac{1}{R}$. If $x \in \partial D$ then there is a ball of radius $R$ tangent to $x$ and contained in $D$. This is a classical result of Blaschke [Bl]. We can view Blaschke's theorem in terms of the minimum distance from $\partial D$ to its interior cut locus. In these terms the result says that for convex domains in $\mathbb{R}^{n}$ a positive lower bound for this distance can be given once we have appropriate bounds on the curvature of the boundary $\partial D$. That things are not so simple for boundary convex domains in a general manifold is clear once we consider the domain on an infinite cylinder $S^{1} \times R^{1}$ bounded by two geodesic circles a distance $\delta$ apart. Although the curvature of the boundary of such a domain is zero, we cannot guarantee that it will contain a ball of a given size. There even exist counterexamples 
in spaces of negative curvature where the domain is contractable. The final section of this paper gives results that show how far the result of Blaschke quoted above can be generalized to domains in manifolds. That there must be exceptional cases is made clear by the example of a ring on a cylinder given above. For domains in manifolds with nonnegative sectional curvature the exceptional cases are shown to have a simple structure not unlike the ring example given here.

Some of the results of this paper and those of [D-L] require that some kind of convexity condition on $D$ holds. We give the relevant definitions. Given a submanifold $N$ of codimension 1 in $M$, the shape operator with respect to a (local) unit normal field $\nu$ on $N$ is defined by

$$
S_{\nu}^{N}(u) \equiv \nabla_{u} \nu
$$

for $u \in T N$. We need only have $\nu$ smoothly defined along a curve with tangent $u$. We will be concerned mainly with the case for which $N$ is $\partial D$ or an inner parallel set for a portion of $\partial D$. Let $\nu_{\text {in }}$ denote the inward normal field to $\partial D$.

DEFINITION. We will call a domain $D$ in a Riemannian manifold boundary-convex if $-\left\langle S_{\nu_{\mathrm{in}}}^{\partial D}(u), u\right\rangle \geq 0$ for all $u \in T \partial D$. We write $0 \leq-S_{\nu_{\text {in }}}^{\partial D}$ to express this.

We use this terminology to distinguish this type of convexity from the idea of geodesically convex.

Definition. A domain $D$ in a Riemannian manifold is called geodesically convex if every two of its points are connected by a minimizing geodesic contained completely within $D$. If the minimizing geodesic is unique we call the set strongly convex.

Definition. $D$ is weakly convex (in the sense of [D-L]) if whenever two points in $\partial D$ are connected by a unique minimizing geodesic segment then that segment is contained in the closure of $D$.

For domains with smooth boundary it is true that all strongly convex domains are geodesically convex, all geodesically convex domains are weakly convex, and all weakly convex domains are boundary convex.

2. Eigenvalues, the heat kernel, and geometry. If $M$ is noncompact, then assume $D \subset B(p, R)$, a geodesic ball of fixed radius $R$. For convenience of notation, set $R=\operatorname{diam} M+1$, when $M$ is compact. Thus $R$ is an upper bound for the out-radius of $D$. Finally, we 
assume that we are given a number $\varrho$ such that $\exp _{\partial D}^{\perp}: \nu(\partial D) \rightarrow M$ is a diffeomorphism in a tube $\Gamma=\{v \in \nu(\partial D)|| v \mid<\varrho\}$. This means that $\varrho$ is a lower bound for $c_{\partial D}$.

Let $\varrho_{1}$ be such that $(r, p) \mapsto \exp _{\perp}^{\partial D} r \nu_{\text {in }}(p)$ is a diffeomorphism on $\left(0, \varrho_{1}\right) \times \partial D$. Thus $\varrho_{1}$ gives the size of an inner tube. Similarly let $\varrho_{2}$ give the width of an outer tube. For weakly convex domains such a $\varrho_{2}$ can be determined solely from the geometry of $M$. This is shown in [D-L]. We may as well assume that $\varrho=\min \left\{\varrho_{1}, \varrho_{2}\right\}$. In all that follows we will assume that $M$ has positive injectivity radius $i_{M}$. It should also be noted that when $i_{M}$ appears below it may be replaced by simply a positive lower bound for $i_{M}$.

REMARK. Most of the results in this section involve $\varrho_{1}$ as given in the hypotheses. However, for suitably convex domains in manifolds of nonnegative curvature, $\varrho_{1}$ can be estimated in terms of the geometry of the ambient manifold and an upper bound on the principal curvatures of the boundary of the domain. This is done in $\S 3$. For this reason each of the main results of this section gives rise to a corresponding result for convex domains where the reference to $\varrho_{1}$ is dropped from the hypothesis while assumptions on the boundary curvature are included. Actually, for a certain class of exceptional domains, which we identify in $\S 3$, we also need to know $\lambda_{1}$ to get the needed estimate on $\varrho_{1}$. This is clearly not a drawback when applied to the results of this section since we use a finite number of eigenvalues anyway. The way in which $\lambda_{1}$ comes in to play is explained near the end of $\S 3$.

LEMMA 2.1. (i) Let $D \subset M$ have a proper inner tube of width $\varrho_{1}$ as described above. Then we have $h \leq S_{\nu_{\text {in }}}^{\partial D}$ where $h$ depends only on $n, \varrho_{1}, i_{M}$, and a lower bound $-a$ for the sectional curvature of $M$. We also have $\bar{h} \leq \operatorname{trace} S_{\nu_{\text {in }}}^{\partial D}$ where $\bar{h}$ depends only on $n, \varrho_{1}, i_{M}$, and a lower bound $-(n-1) a$ for the Ricci curvature of $M$.

(ii) Let $D \subset M$ have a proper outer tube of width $\varrho_{2}$ as described above. Then we have $H \geq S_{\nu_{\text {in }}}^{\partial D}$ where $H$ depends only on $n, \varrho_{2}, i_{M}$, and a lower bound - a for the sectional curvature of $M$. We also have $\bar{H} \geq \operatorname{trace} S_{\nu_{\text {in }}}^{\partial D}$ where $\bar{H}$ depends only on $n, \varrho_{2}, i_{M}$, and a lower bound - $(n-1)$ a for the Ricci curvature of $M$.

Proof. (i) First assume $\varrho_{1} \leq i_{M}$. There is a ball $B$ of radius $\varrho_{1} / 2$ contained inside $D$ with center a distance $\varrho_{1} / 2$ from a given point 
$p \in \partial D$. Now, $\partial B$ is smooth at $p$ and tangent to $\partial D$ at $p$ and thus

$$
S_{\bar{\nu}_{\text {in }}}^{\partial B}(p) \leq S_{\nu_{\mathrm{in}}}^{\partial D}(p),
$$

where $\bar{\nu}_{\text {in }}$ is the inward normal for $\partial B$. If $-a$ is a lower bound for the sectional curvature then by comparing with a ball of radius $\varrho_{1} / 2$ in a hyperbolic space of constant curvature $-a$ (or a Euclidean space if $a=0$ ) we have

$$
h \leq S_{\bar{\nu}_{\text {in }}}^{\partial B},
$$

where $h$ depends on $\varrho_{1}, n$, and $a$. If we are only given a lower bound on the Ricci curvature we can only conclude that

$$
\bar{h} \leq \operatorname{trace} S_{\bar{\nu}_{\text {in }}}^{\partial B} \text {. }
$$

The first result follows. For the general case we just replace $\varrho_{1}$ by $\min \left\{\varrho_{1}, i_{M}\right\}$.

(ii) The proof of (ii) is analogous.

LEMMA 2.2. Let $D$ be a domain with smooth boundary in $M$.

(i) Let $-a(n-1)$ be a lower bound on the Ricci curvature of $M$ where $a \geq 0$. Then vol $D \leq c_{1}$ where $c_{1}$ depends only on $n, a$, and $R$.

(ii) Let - $a$ be a lower bound on the sectional curvature of $M$ and $b$ an upper bound for the sectional curvature of $M$, where $a, b \geq 0$. Then vol $\partial D \leq c_{2}$ where $c_{2}$ depends only on $n, R, a, b, i_{M}$, and either $\varrho_{1}$ or $\varrho_{2}$.

Proof. The proof of (i) is immediate since $\operatorname{vol} D \leq \operatorname{vol} B(p, R+1)$ $\leq \operatorname{vol} B_{-a}(R+1)$, where $B_{-a}(R+1)$ is a ball of radius $R+1$ in the simply connected hyperbolic space of constant curvature $-a$ if $-a<0$, and in $\mathbb{R}^{n}$ if $a=0$. This last inequality is Bishop's volume comparison for balls.

For the proof of (ii) we first consider the case where we are given $\varrho_{1}$. Let $\Gamma_{1}$ denote the inner proper tube of width $\varrho_{1}$ on which $\exp _{\perp}^{\partial D}$ is a diffeomorphism. By the comparison theory of $[\mathbf{H}-\mathbf{K}]$ or $[\mathbf{W}]$ we can find a $c$ depending only on $n, \varrho_{1}, b$, and a lower bound for $S_{\nu_{\text {in }}}^{\partial D}$ such that

$$
\operatorname{vol}(\partial D) \leq c \operatorname{vol} \Gamma_{1} .
$$

But by Lemma 2.1(i) we can take $c$ to depend only on $n, \varrho_{1}, i_{M}$, $b$, and $a$. Now vol $\Gamma_{1} \leq \operatorname{vol} B(p, R+1)$ for some $p \in D$ and $\operatorname{vol} B(p, R+1) \leq \operatorname{vol} B_{-a}(R+1)$ where $B_{-a}(R+1)$ is a ball of radius 
$R+1$ in the simply connected hyperbolic space of constant curvature $-a$ if $-a<0$ and in $\mathbb{R}^{n}$ if $a=0$. The result in the case where we are given $\varrho_{1}$ follows. The case where we are given $\varrho_{2}$ is proved similarly using an outer tube and Lemma 2.1(ii).

THEOREM 2.3. Let $M$ be a (possibly noncompact) complete Riemannian manifold with sectional curvature $K$ and positive injectivity radius $i_{M}$. Let $D$ be a bounded domain with smooth compact boundary. Suppose also that $-a \leq K \leq b$, where $a, b \geq 0$. Then given $0<\delta<1$ there is a number $N(\delta)$ such that

$$
\left|(4 \pi \delta)^{n / 2} \sum_{i=1}^{N(\delta)} e^{-\lambda_{i} \delta}-\operatorname{vol}(D)\right| \leq c \delta^{1 / 2},
$$

where $N(\delta)$ and $c$ depend only on $n, \varrho_{2}, a, b, i_{M}$, and $R$.

Proof. Apply Theorem 3.5 of [D-L] . This reads

$$
\begin{aligned}
& \left|(4 \pi t)^{n / 2} \operatorname{Tr} E_{D}(t)-\operatorname{vol} D\right| \\
& \leq \alpha_{1}\left(\int_{0}^{\infty} e^{\alpha_{2} s} e^{-\alpha_{3} s^{2} / a t} d s\right) \operatorname{vol}(\partial D) C \\
& \quad+\alpha_{4}(a+b) t \operatorname{vol}(D)+d t \operatorname{vol}(D) t^{n / 2}
\end{aligned}
$$

where $E_{D}$ is the heat kernel for $D$ with Dirichlet boundary conditions. Also, $\alpha_{1}, \alpha_{2}, \alpha_{3}$ and $\alpha_{4}$ are universal constants and $d$ depends only on $b$ and the injectivity radius $i_{M}$ of $M$ and $C$ depends on $a$ and some upper bound for the mean curvature trace $S_{\nu_{\text {in }}}^{\partial D}$. But, by Lemma 2.1(ii), this upper bound can be given in terms of $n$, $a, \varrho_{2}$, and $i_{M}$. By Lemma 2.2 we see that the right-hand side of (2.4) is less than $C_{3} t^{1 / 2}$ for $0<t<1$, where $C_{3}$ depends only on $n, a, b, i_{M}, \varrho_{2}$, and $R$. Now, by a well known result [D-Li], there exist constants $m$ and $C_{5}$ so that $\lambda_{i}(D) \geq C_{5} i^{2 / n}$ for $i \geq m$ and where $m$ and $C_{5}$ depend only on $n, a, b, i_{M}$, and $R$.

Now choose $N(\delta) \geq m$ so that

$$
\sum_{i=N(\delta)+1}^{\infty} e^{-\lambda_{l} \delta} \leq \sum_{i=N(\delta)+1}^{\infty} e^{-C_{5} i^{2 / n} \delta}<C_{6} \delta^{1 / 2}
$$


Then we have

$$
\begin{aligned}
& \left|(4 \pi \delta)^{n / 2} \sum_{i=1}^{N(\delta)} e^{-\lambda_{i} \delta}-\operatorname{vol}(D)\right| \\
& \leq C_{6} \delta^{1 / 2}+\left|(4 \pi \delta)^{n / 2} \operatorname{Tr} E_{D}(\delta)-\operatorname{vol}(D)\right| \\
& \quad \leq\left(C_{6}+C_{3}\right) \delta^{1 / 2}
\end{aligned}
$$

Theorem 2.3 above should be compared to Theorem 4.3 of [D-L]. The difference is that in Theorem 2.3 we do not need to have $D$ weakly convex and we need only the injectivity radius as opposed to the convexity radius of $M$. However, we do need to know the bound $\varrho_{2}$ which was not needed in [D-L].

Next we study the role of the boundary. In all statements below, $c_{i}$ denote constants depending upon just $R, \varrho$, and the geometry of $M$ unless otherwise stated. By the geometry of $M$ we mean $\operatorname{dim} M=n$, an upper bound $b$ and a lower bound $-a$ on the sectional curvature of $M$, and a lower bound on its injectivity radius. Here we take $a, b \geq$ 0 . We may suppose that $\varrho \leq 1$ and that $\varrho$ is less than the injectivity radius of $B(p, R+2)$. Let $A$ be a domain with smooth boundary satisfying $B(p, R+2) \subseteq A \subseteq B(p, R+3)$. Consider a point $x \in D$ of distance $d(x) \leq \varrho / 2$ from $\partial D$. The point, in $\partial D$, which is closest to $x$ will be denoted as $y$. There are balls $B$ and $B_{1}$, each of radius $\varrho / 2$, which are respectively internally and externally tangent, at $y$, to $\partial D$. Let $C=A-B_{1}$. We have that $B \subset D \subset C$. Consequently, Duhamel's principle gives the monotonicity of heat kernels:

$$
E_{B}(x, x, t) \leq E_{D}(x, x, t) \leq E_{C}(x, x, t) .
$$

It follows from the standard parametrix construction [M-S, R-S] of the heat kernel, carried out in harmonic coordinates, that

$$
\begin{gathered}
\max \left\{\left|E_{B}(x, x, t)-(4 \pi t)^{-n / 2}\left(1-e^{-d^{2}(x) / t}\right)\right|,\right. \\
\left.\left|E_{C}(x, x, t)-(4 \pi t)^{-n / 2}\left(1-e^{-d^{2}(x) / t}\right)\right|\right\} \\
\leq c_{4} e^{-d^{2}(x) / 4 t} t^{-n / 2+1 / 2}+c_{5} t^{-n / 2+1}+c_{6} t .
\end{gathered}
$$

Combining (2.5)and (2.6), we get, for $d(x) \leq \varrho / 2$,

$$
\begin{aligned}
& \mid E_{D}(x, x, t)-(4 \pi t)^{-n / 2}\left(1-e^{-d^{2}(x) / t}\right) \mid \\
& \leq c_{4} e^{-d^{2}(x) / 4 t} t^{-n / 2+1 / 2}+c_{5} t^{-n / 2+1}+c_{6} t .
\end{aligned}
$$


If $d(x) \geq \varrho / 2$, then from [D-L], one has

$$
\left|E_{D}(x, x, t)-(4 \pi t)^{-n / 2}\right| \leq c_{7} t^{-n / 2+1}+c_{8} t .
$$

It is now easy to derive a remainder estimate for $E_{D}$. If $M=R^{n}$, this was proved earlier by van den Berg [B]. One has

THEOREM 2.9.

$$
\left|\operatorname{Tr} E_{D}(t)-\frac{\operatorname{vol} D}{(4 \pi t)^{n / 2}}+\frac{\operatorname{vol} \partial D}{4(4 \pi t)^{(n-1) / 2}}\right| \leq c_{9} t^{-n / 2+1}+c_{10} t .
$$

Proof. One computes the trace of the heat kernel by integrating its restriction to the diagonal:

$$
\operatorname{Tr} E_{D}(t)=\int_{D} E_{D}(x, x, t) d x .
$$

Let $W$ denote the set where $d(x) \leq \varrho / 2$. Then

$$
\operatorname{Tr} E_{D}(t)=\int_{W} E_{D}(x, x, t) d x+\int_{D-W} E_{D}(x, x, t) d x .
$$

Using (2.8) and Lemma 2.2, we may write

$$
\begin{gathered}
\left|\int_{D-W} E_{D}(x, x, t)-(4 \pi t)^{-n / 2} \operatorname{vol}(D-W)\right| \\
\leq c_{11} t^{-n / 2+1}+c_{12} t .
\end{gathered}
$$

Similarly from (2.7) and Lemma 2.2,

$$
\begin{gathered}
\left|\int_{W} E_{D}(x, x, t)-(4 \pi t)^{-n / 2} \operatorname{vol} W+(4 \pi t)^{-n / 2} \int_{W} e^{-d^{2}(x) / t}\right| \\
\leq c_{13} t^{-n / 2+1}+c_{14} t .
\end{gathered}
$$

Comparison theory, for Jacobi fields along $\partial D,[\mathbf{H}-\mathbf{K}]$, and the upper bound of $\operatorname{vol}(\partial D)$, were employed in the most recent estimate.

Invoking comparison principles again, along with the elementary formula, [S], $\int_{0}^{\infty} e^{-s^{2}} d s=\sqrt{\pi} / 2$, gives

$$
\begin{aligned}
\mid \int_{W} E_{D}(x, x, t)- & (4 \pi t)^{-n / 2} \operatorname{vol} W+\frac{\operatorname{vol} \partial D}{4(4 \pi t)^{n-1 / 2}} \mid \\
& \leq c_{17} t^{-n / 2+1}+c_{18} t
\end{aligned}
$$

Theorem 2.9 follows by combining (2.10), (2.11), and (2.12).

One may apply Theorem 2.9 to inverse spectral theory. In Theorem 2.3 we determined vol $D$ up to arbitrary accuracy from a finite part of the spectrum of $\Delta$. It is now possible to derive a similar result for 
$\operatorname{vol} \partial D$ :

THEOREM 2.13. Let $D$ be a bounded domain with smooth boundary in $M$. Let $-a$ be a lower bound on the sectional curvature of $M$ and $b$ an upper bound for the sectional curvature of $M$, where $a, b \geq 0$. For any given $\varepsilon>0$, there exists a constant $c_{19}$, depending upon $\varepsilon$, so that: $\operatorname{vol}(\partial D)$ is determined, up to error of order $\varepsilon$, by those $\lambda_{i}$ with $i \leq c_{19}$. The number $c_{19}$ depends only on $\varepsilon, a, b, i_{M}, R$, and $\varrho$.

Proof. From Theorem 2.9, we may choose $\delta$ suitably small, so that $\left|4(4 \pi \delta)^{\frac{n-1}{2}} \operatorname{Tr} E_{D}(\delta)-\frac{2 \operatorname{vol} D}{(\pi \delta)^{1 / 2}}+\operatorname{vol} \partial D\right| \leq c_{20} \delta^{1 / 2}<\varepsilon / 3$.

Fix such a choice of $\delta$.

By Theorem 2.3, knowledge of the $\lambda_{i}$, for $i \leq c_{21}$, determines vol $D$ up to error $\frac{1}{2}(\pi \delta)^{\frac{1}{2}} \varepsilon / 3$. Let $\operatorname{vol}_{\varepsilon} D$ be such an approximate value for vol $D$. Then

$$
\left|4(4 \pi \delta)^{\frac{n-1}{2}} \operatorname{Tr} E_{D}(\delta)-\frac{2 \operatorname{vol}_{\varepsilon} D}{(\pi \delta)^{1 / 2}}+\operatorname{vol} \partial D\right| \leq 2 \varepsilon / 3 .
$$

Recall that $\operatorname{Tr} E_{D}(\delta)=\sum_{i=1}^{\infty} e^{-\delta \lambda_{i}}$. As shown in the proof of Theorem 2.3, we can choose $c_{22}$ so that

$$
4(4 \pi \delta)^{\frac{n-1}{2}} \sum_{i=c_{22}}^{\infty} e^{-\delta \lambda_{i}} \leq \varepsilon / 3 .
$$

Combining these ingredients gives for $c_{19} \geq \max \left(c_{21}, c_{22}\right)$ :

$$
\left|4(4 \pi \delta)^{\frac{n-1}{2}} \sum_{i=1}^{c_{19}} e^{-\delta \lambda_{i}}-\frac{2 \operatorname{vol}_{\varepsilon} D}{(\pi \delta)^{1 / 2}}+\operatorname{vol} \partial D\right| \leq \varepsilon .
$$

Since $\operatorname{vol}_{\varepsilon} D$ is determined by the first $c_{21}$ eigenvalues, the proof of Theorem 2.13 is complete.

We may estimate the out-radius for geodesically convex domains. Let $\mathscr{M}(\cdot)$ be the eigenvalue counting function as in Theorem 1.1.

Theorem 2.14. Let $D \subset M$ be a geodesically convex domain with smooth boundary. Assume that the Ricci curvature of $M$ satisfies $-a(n-1)|X|^{2} \leq \operatorname{Ric}(X, X), a \geq 0$, for all $X \in T M$. Let $\varrho_{1}$ be given as above. Then

$$
R_{\text {out }} \leq 4 \varrho_{1}\left(\mathscr{M}\left(\beta\left(n, a, \varrho_{1}\right)+1\right)\right)
$$

where $\beta\left(n, a, \varrho_{1}\right)$ depends only on $a, \varrho_{1}$, and $\operatorname{dim} M=n$. 
Proof. Let $B_{0}$ be a ball of radius $\varrho_{1}$ with center $p_{0}$, for $p_{0} \in D$. We take $p_{0}$ to be a distance $\varrho_{1}$ from $\partial D$ and then it is not hard to see from the definition of $\varrho_{1}$ that $B_{0} \subset D$. Let $q$ be the furthest point from $p_{0}$ in $\bar{D}$. We have that $R_{\text {out }} \leq \operatorname{dist}\left(p_{0}, q\right)$, where dist denotes the geodesic distance. Let $\gamma:[0,1] \rightarrow \bar{D}$ be a minimizing geodesic from $p_{0}=\gamma(0)$ to $q=\gamma(1)$. Let $k \geq 0$ be the largest integer such that there exist $t_{0}, t_{1}, \ldots, t_{k}$ with $0=t_{0} \leq t_{1}<t_{2}<\cdots<t_{k} \leq 1$ and with $\operatorname{dist}\left(\gamma\left(t_{i}\right), \gamma\left(t_{i+1}\right)\right)=4 \varrho_{1}$.

We have

$$
k \geq\left[\frac{\operatorname{dist}\left(p_{0}, q\right)}{4 \varrho_{1}}\right] \geq \frac{R_{\text {out }}}{4 \varrho_{1}}-1 .
$$

Now if $\operatorname{dist}\left(\gamma\left(t_{i}\right), \partial D\right) \geq \varrho_{1}$ then let $B_{i} \equiv B\left(\gamma\left(t_{i}\right), \varrho_{1}\right)$. If $\operatorname{dist}\left(\gamma\left(t_{i}\right), \partial D\right)<\varrho_{1}$, let $x_{i}$ be the closest point of $\partial D$ to $\gamma\left(t_{i}\right)$ and let $B_{i}$ be the ball of radius $\varrho_{1}$ centered at $\exp _{\perp}^{\partial D} \varrho_{1} \nu_{\text {in }}\left(x_{i}\right)$. We have that $\gamma\left(t_{i}\right)=\exp _{\perp}^{\partial D} r \nu_{\text {in }}\left(x_{i}\right)$ for some $0 \leq r<\varrho_{1}$ and hence the distance from $\gamma\left(t_{i}\right)$ to the center of $B_{i}$ is less than $\varrho_{1}$. The triangle inequality gives that $B_{i} \cap B_{j}=\varnothing$ for $i \neq j$. Also $B_{i} \subset D$ for $0 \leq i \leq k$.

Let $\mathbb{H}_{-a}^{n}$ be the simply connected space of constant curvature $-a \leq$ 0 and dimension $n$, let $B_{-a}(r)$ be a geodesic ball of radius $r$ in that space, and $\lambda_{1}\left(B_{-a}(r)\right)$ its first Dirichlet eigenvalue. Choose

$$
\beta\left(n, a, \varrho_{1}\right) \geq \lambda_{1}\left(B_{-a}\left(\varrho_{1}\right)\right) .
$$

Note that $\beta\left(n, a, \varrho_{1}\right)$ may be chosen explicitly by using the upper bounds for $\lambda_{1}\left(B_{-a}(r)\right)$ given for example in [Ga]. In particular if $a=0$ we may let $\beta=n(n+4) / 2 \varrho_{1}^{2}$ as in [T]. We now show that $k \leq \mathscr{M}\left(\beta\left(n, a, \varrho_{1}\right)\right)$. The Poincaré minimum principle asserts that

$$
\lambda_{k}(D)=\inf \frac{\int_{D}|d f|^{2}}{\int_{D} f^{2}},
$$

where the infimum is taken over all $f \in H_{0}^{1}$ such that $f$ is $L^{2}$ orthogonal to the eigenfunctions corresponding to $\lambda_{1}, \ldots, \lambda_{k-1}$. Let $f^{i}$ be the first eigenfunction of $B_{i}$. We can choose constants $a_{i}$ so that $\sum a_{i} f^{i}$ satisfies the orthogonality conditions needed for the minimum principle. Then we have

$$
\begin{aligned}
\lambda_{k}(D) & \leq \frac{\int_{D}|d f|^{2}}{\int_{D} f^{2}} \leq \frac{\sum a_{i}^{2} \int_{B_{i}}\left|d f^{i}\right|^{2}}{\sum a_{i}^{2} \int_{B_{i}}\left(f^{i}\right)^{2}} \\
& \leq \sup _{i} \frac{\int_{B_{i}}\left|d f^{i}\right|^{2}}{\int_{B_{i}}\left(f^{i}\right)^{2}} \leq \sup _{i} \lambda_{1}\left(B_{i}\right) \leq \lambda_{1}\left(B_{-a}\left(\varrho_{1}\right)\right),
\end{aligned}
$$


where we have used Cheng's eigenvalue comparison [C]. Thus, by the definition of $\mathscr{M}$, we have $k \leq \mathscr{M}\left(\beta\left(n, a, \varrho_{1}\right)\right)$. Hence, $\frac{R_{\text {out }}}{4 \varrho_{1}}-1 \leq$ $\mathscr{M}\left(\beta\left(n, a, \varrho_{1}\right)\right)$ which implies the result.

The following theorem should be compared to Lemma 2.2. Both results give upper bounds for vol $\partial D$ and vol $D$. We point out some of the differences. First, the constants of Lemma 2.2 depend on $R$, and $\varrho_{1}$ or $\varrho_{2}$ while in Theorem 2.15 we need only $\varrho_{1}$. Secondly, we point out that the upper bounds below involve the counting function $\mathscr{M}$ and thus a finite part of the spectrum becomes involved.

THEOREM 2.15. Let $D$ be a domain with compact smooth boundary in a complete Riemannian manifold $M$. Assume that the sectional curvature satisfies $-a \leq K \leq b$, where $a, b \geq 0$. In particular, $D$ might not be convex. If $\varrho_{1}$ is a lower bound on the distance from $D$ to the interior part of the cut locus of $\partial D$ as before then

$$
\operatorname{vol}(\partial D) \leq C\left(n, \varrho_{1}, i_{M}, a, b\right) \mathscr{M}\left(\beta_{1}\left(n, a, \varrho_{1}\right)\right) .
$$

If we weaken the lower bound on sectional curvature to a lower bound on Ricci curvature, $-a(n-1) \leq$ Ric, then

$$
\operatorname{vol}(D) \leq C_{1}\left(n, \varrho_{1}, a\right) \mathscr{M}\left(\beta_{1}\left(n, a, \varrho_{1}\right)\right) .
$$

The constants depend only on the indicated quantities. Also, $C$ above may be taken to depend on a lower bound for $S_{\nu_{\mathrm{in}}}^{\partial D}$ instead of $i_{M}$ and in this case we can take the lower bound on curvature to be the Ricci curvature bound $-a(n-1) \leq$ Ric.

Proof. Let $\Gamma$ be the region inside $\bar{D}$ given by

$$
\Gamma=\left\{\exp _{\perp}^{\partial D}\left(r \nu_{\text {in }}(p)\right): p \in \partial D, 0 \leq r \leq \frac{\varrho_{1}}{2}\right\}
$$

Now, fit as many balls $B_{i}\left(\varrho_{1} / 4\right)$ inside $\Gamma$ as possible without intersecting interiors and with centers a distance $\varrho_{1} / 4$ from $\partial D$. Let this number be $N$. Define $\rho$ on $\nu(\partial D)$ by $\rho(\xi)=\langle\xi, \xi\rangle^{1 / 2}$ for $\xi \in \nu(\partial D)$. Now using the fact that for points inside $\Gamma$ the distance to the boundary is given by $\rho \circ \exp _{\perp}^{-1}$, and using the triangle inequality, we see easily that a ball of radius $\varrho_{1} / 4$ centered at a point in $D$ at a distance $\varrho_{1} / 4$ from the boundary $\partial D$ is contained completely inside $\Gamma$. It follows from the maximality of $N$ that every point of $\Gamma$ is at most $3 \varrho_{1} / 4$ from the center of some $B_{i}$. Thus, by Bishop's 
volume comparison for balls,

$$
\operatorname{vol}(\Gamma) \leq N \operatorname{vol} B_{-a}\left(\frac{3 \varrho_{1}}{4}\right)
$$

Note that so far we only need the lower bound on Ricci curvature. Now by the comparison theory of $[\mathbf{H}-\mathbf{K}]$ again we can find a $c$ depending only on $n, \varrho_{1}, b$, and a lower bound for $S_{\nu_{\text {in }}}^{\partial D}$ such that

$$
\operatorname{vol}(\partial D) \leq c \operatorname{vol} \Gamma \text {. }
$$

But now if $-a$ is taken as a lower bound on sectional curvature then by Lemma 2.1 we can take $c$ to depend only on $n, \varrho_{1}, i_{M}$, and $a$. Hence,

$$
\operatorname{vol}(\partial D) \leq N c \operatorname{vol} B_{-a}\left(\frac{3 \varrho_{1}}{4}\right) .
$$

Let $\beta_{1}\left(n, a, \varrho_{1}\right) \geq \lambda_{1}\left(B_{-a}\left(\varrho_{1} / 4\right)\right)$. As in the proof of Theorem 2.14 we can use the Poincaré minimum principle to show that

$$
\lambda_{N}(D) \leq \lambda_{1}\left(B_{-a}\left(\frac{\underline{\varrho}_{1}}{4}\right)\right) .
$$

Thus, $N \leq \mathscr{M}\left(\beta_{1}\left(n, a, \varrho_{1}\right)\right)$. Finally, the above inequalities combine to give

$$
\operatorname{vol}(\partial D) \leq C\left(n, \varrho_{1}, i_{M}, a, b\right) \mathscr{M}\left(\beta_{1}\left(n, a, \varrho_{1}\right)\right)
$$

Now we derive the volume inequality. We only need the lower bound on Ricci curvature. In addition to the $N$ balls of radius $\varrho_{1} / 4$ inside $\Gamma$, fit as many disjoint balls $B_{j}^{\prime}$ of radius $\varrho_{1} / 4$ inside $D-\Gamma$ as possible. Call this number $N^{\prime}$. Every point of $D-\Gamma$ must be either at a distance of not greater than $\varrho_{1} / 2$ from the center of one of the $B_{j}^{\prime}$ or at a distance of not greater than $\varrho_{1} / 4$ from $\Gamma$ since otherwise we could fit another ball of radius $\varrho_{1} / 4$ into $D-\Gamma$. Since a point that has distance less than or equal to $\varrho_{1} / 4$ of $\Gamma$ is within a distance $\varrho_{1}$ of the center one of the $B_{i} \subset \Gamma$, we see that the $N+N^{\prime}$ balls say $\widetilde{B}_{i}$ and $\widetilde{B}_{i}^{\prime}$, with centers coinciding with those of the $B_{i}$ and $B_{j}^{\prime}$ but with radii $\varrho_{1}$, cover $D$. Hence, we have

$$
\operatorname{vol}(D) \leq\left(N+N^{\prime}\right) \sup _{i, j}\left\{\operatorname{vol}\left(\widetilde{B}_{i}\right), \operatorname{vol}\left(\widetilde{B}_{j}^{\prime}\right)\right\} \leq\left(N+N^{\prime}\right) \operatorname{vol}\left(B_{-a}\left(\varrho_{1}\right)\right) .
$$

Now, arguing with the minimum principle as before, we easily obtain

$$
\lambda_{N+N^{\prime}}(D) \leq \lambda_{1}\left(B_{-a}\left(\frac{\varrho_{1}}{4}\right)\right) .
$$

Thus, as before, we can conclude that

$$
N+N^{\prime} \leq \mathscr{M}\left(\beta_{1}\left(n, a, \varrho_{1}\right)\right)
$$


and the above combine to give the second inequality of the theorem. To get the final statement we simply forego the use of Lemma 2.1.

Theorems 2.16 and 2.17 below should be compared to Theorems 2.3 and 2.13. Notice that the out-radius bound $R$ makes no appearance in the results which follow.

THEOREM 2.16. Let $M$ be a (possibly noncompact) complete Riemannian manifold with sectional curvature $K$ and positive injectivity radius $i_{M}$. Let $D$ be a bounded connected domain with smooth boundary. Suppose also that $-a \leq K \leq b$, where $a, b \geq 0$. Then given $0<\delta<1$ there is a number $N(\delta)$ such that

$$
\left|(4 \pi \delta)^{n / 2} \sum_{i=1}^{N(\delta)} e^{-\lambda_{t} \delta}-\operatorname{vol}(D)\right| \leq c \delta^{1 / 2}
$$

where $N(\delta)$ and $c$ depend only on $n, \varrho, a, b, i_{M}$ and $\mathscr{M}\left(\beta_{1}\left(n, a, \varrho_{1}\right)\right)$.

Also, if we are given an upper bound $h_{2}$ for the mean curvature trace $S_{\nu_{\mathrm{n}}}^{\partial D}$ then we may replace $\varrho$ by $\varrho_{1}$ and $h_{2}$.

Proof. The proof is like that of Theorem 2.3 except that we use Theorem 2.15 instead of Lemma 2.2. To get the final statement we simply forego the use of Lemma 2.1(ii).

THEOREM 2.17. Let $D$ be a bounded domain with smooth boundary in $M$. Let $-a$ be a lower bound on the sectional curvature of $M$ and $b$ an upper bound for the sectional curvature of $M$ where $a, b \geq 0$. For any given $\varepsilon>0$, there exists a constant $C_{19}$, depending upon $\varepsilon$, so that: $\operatorname{vol}(\partial D)$ is determined, up to error of order $\varepsilon$, by those $\lambda_{i}$ with $i \leq C_{19}$. The constant $C_{19}$ depends only on $\varepsilon, n, \varrho, a, b, i_{M}$, and $\mathscr{M}\left(\beta_{1}\left(n, a, \varrho_{1}\right)\right)$.

Proof. The proof follows the proof of Theorem 2.13 except that we use Theorem 2.15 instead of Lemma 2.2. Also Theorem 2.16 is cited to replace Theorem 2.3.

3. $\varrho_{1}$ and the geometry of convex domains. We now show how to estimate $\varrho_{1}$ in terms of the geometry of $M$ and the boundary curvature of $D$. We have already used the submanifold comparison theory found for example in $[\mathbf{H}-\mathbf{K}],[\mathbf{G}]$ and [W]. In what follows we will need a part of these results which we now explain. Let $D$ 
be a domain in a Riemannian manifold $M$ of dimension $n$. We assume throughout that $\partial D$ is smooth and compact. Let $K$ denote the sectional curvature of $M$. If $N$ is any regular submanifold of $M$, let $\pi: \nu(N) \rightarrow N$ denote the associated normal bundle. Given a curve $\xi:(-\varepsilon, \varepsilon) \rightarrow \nu(N)$ with $\|\xi(t)\|=1$ for all $t \in(-\varepsilon, \varepsilon)$ and letting $\gamma=\pi \circ \xi, \gamma(0)=p, \gamma^{\prime}(0)=u \in T_{p} N$ we can define an $N$-Jacobi field by:

or

$$
Y(s)=\left.\frac{d}{d t} \exp _{\perp}^{N} s \xi(t)\right|_{t=0}
$$

$$
Y(s)=d \exp _{\perp}^{N} U(s)
$$

where

$$
U(s)=\left.\frac{d}{d t} s \xi(t)\right|_{t=0} .
$$

A short calculation gives

$$
\left.\frac{d}{d s} \log \|Y(s)\|\right|_{s=0}=\frac{\left\langle S_{\xi}^{N}(u), u\right\rangle}{\|u\|^{2}}
$$

where $\xi=\xi(0)$. Let $\nu$ be a unit normal field for $N$. In case $N=\partial D$ we agree that $\nu=\nu_{\text {in }}$ and for a point in question $p \in \partial D$ and $u \in T_{p} \partial D$ that $\xi$ be defined by $\xi=\nu_{\text {in }} \circ \gamma$ where $\gamma$ is some curve in $\partial D$ with $\gamma(0)=p$ and $\gamma^{\prime}(0)=u$. Of course, the $N$-Jacobi fields $Y(s)$ depend on the choice of $p$ and $u$ but this will remain tacit for notational brevity.

Let $s_{1}, s_{2}, \ldots, s_{n-1}$ denote the eigenvalues of $S_{\nu}^{N}$. Now, according to the comparison theory of $[\mathbf{W}]$ or $[\mathbf{H}-\mathbf{K}]$ if we have another situation where we are given a manifold $\bar{M}$ with sectional curvature $\bar{K}$, a submanifold $\bar{N}$, and associated $\bar{U}(s), \bar{Y}(s), \bar{s}_{i}$ etc. then we have the following:

I. If $\inf _{M} K \geq \bar{K}$ and $\max _{i}\left\{s_{i}\right\} \leq \min _{i}\left\{\bar{s}_{i}\right\}$ then

$$
\frac{\|Y(s)\|}{\|U(s)\|} \leq \frac{\|\bar{Y}(s)\|}{\|\bar{U}(s)\|}
$$

and

$$
\frac{d}{d s} \log \|Y(s)\| \leq \frac{d}{d s} \log \|\bar{Y}(s)\|
$$

for all nonnegative $s$ not greater than the first focal distance of $N$ along the curve $s \mapsto \exp _{\perp}^{N} s \xi(0)$.

II. If $\bar{K} \geq \sup _{M} K$ and $\max _{i}\left\{\bar{s}_{i}\right\} \leq \min _{i}\left\{s_{i}\right\}$ then

$$
\frac{\|Y(s)\|}{\|U(s)\|} \geq \frac{\|\bar{Y}(s)\|}{\|\bar{U}(s)\|}
$$


and

$$
\frac{d}{d s} \log \|Y(s)\| \geq \frac{d}{d s} \log \|\bar{Y}(s)\|
$$

for all nonnegative $s$ not greater than the first focal distance of $\bar{N}$ along the curve $s \mapsto \exp _{\perp}^{\bar{N}} s \bar{\xi}(0)$.

Now, if $\exp _{\perp}^{N}$ is a diffeomorphism from a neighborhood $\widetilde{\mathscr{U}}$ in $\nu(N)$ onto $\mathscr{U} \in \mathscr{M}$ then for $\alpha \geq 0$ define

$$
N_{\alpha} \equiv \exp _{\perp}^{N}(\{\alpha \nu(x): x \in N\} \cap \tilde{\mathscr{U}}) .
$$

This is a regular submanifold of codimension 1 whenever it is nonempty. On $\mathscr{U}$ define the function $\left.r \equiv \rho \circ \exp _{\perp}^{N}\right|_{\mathscr{U}} ^{-1}$. Here $N_{\alpha}$ is just the $r=\alpha$ level submanifold. The associated vector field on $\mathscr{U}$ is $\partial_{r}=\operatorname{grad} r$. For nonempty $N_{\alpha}$ we have that $\left.\partial_{r}\right|_{N_{\alpha}}$ is a unit normal field for $N_{\alpha}$. In this case, we also have

$$
\left.\frac{d}{d s} \log \|Y(s)\|\right|_{s=\alpha}=\frac{\left\langle S_{\partial_{r}}^{N_{\alpha}} v, v\right\rangle}{\|v\|^{2}},
$$

where

$$
v=Y(\alpha)
$$

LEMMA 3.5. Let $\widetilde{\mathscr{U}}$ be a connected open set in $\nu(N)$ on which $\exp _{\perp}^{N}$ is a diffeomorphism onto $\mathscr{U}$. Let $N_{\alpha} \subset \mathscr{U}$ and $\partial_{r}$ be as above. Suppose that $N_{\alpha}$ is nonempty and let $L$ be a submanifold of codimension 1 in $\mathscr{U}$ tangent to $N_{\alpha}$ at $q$ and with normal field $\nu$ such that $-\nu=\partial_{r}$ at $q$. Suppose also that $L$ lies in the $r \geq \alpha$ part of $\exp _{\perp}^{N} \mathscr{U}$. Then we have the following.

A. If

$$
S_{\partial_{r}}^{N_{\alpha}}<0
$$

then

$$
-S_{\nu}^{L}<0
$$

at $q$.

B. Assume that L is connected. Suppose that

$$
S_{\partial_{r}}^{N_{\alpha}} \leq 0
$$

and $r(p)>\alpha$ for some $p \in L$. Then there is a point $q_{1} \in L$ such that

$$
\left\langle-S_{\nu}^{L} w, w\right\rangle<0
$$

for some $w \in T_{q_{1}} L$. 
Proof. For A we just take a normal coordinate system $\left\{x^{i}\right\}$ around $q$ with $\frac{\partial}{\partial x^{n}}=\partial_{r}$ at $q$ and $\frac{\partial}{\partial x^{i}}$ tangent to $N_{\alpha}$ at $q$ for $1 \leq i \leq n-1$. Let $\gamma_{1}$ be a curve in $N_{\alpha}$ with $\gamma_{1}^{\prime}(0)=u$ and $\gamma_{2}$ a curve in $L$ with $\gamma_{2}^{\prime}(0)=\gamma_{1}^{\prime}(0)=u$. Now, since the Christoffel symbols vanish at $p$, we have

$$
-\left\langle S_{\partial_{r}}^{N_{\alpha}} \gamma_{1}^{\prime}, \gamma_{1}^{\prime}\right\rangle_{p}=\left\langle\frac{\partial}{\partial x^{n}}, \nabla_{\gamma_{1}^{\prime}} \gamma_{1}^{\prime}\right\rangle_{p}=\left.\frac{d^{2} x^{n}\left(\gamma_{1}(t)\right)}{d t^{2}}\right|_{t=0}
$$

and similarly for $S_{\nu}^{L}$. Thus, the proof reduces to showing that

$$
\left.\frac{d^{2} x^{n}\left(\gamma_{1}(t)\right)}{d t^{2}}\right|_{t=0}>0,\left.\quad \frac{d x^{n}\left(\gamma_{1}(t)\right)}{d t}\right|_{t=0}=\left.\frac{d x^{n}\left(\gamma_{2}(t)\right)}{d t}\right|_{t=0}=0
$$

and $x^{n}\left(\gamma_{2}(t)\right) \geq x^{n}\left(\gamma_{1}(t)\right)$ for small $t$ imply

$$
\left.\frac{d^{2} x^{n}\left(\gamma_{2}(t)\right)}{d t^{2}}\right|_{t=0}>0
$$

which is elementary.

Now we prove B. We have $p \in L$ with $r(p)>\alpha$. Join $p$ to $q$ with a smooth curve in $\mathscr{U} \cap L$ starting at $q=\gamma(0)$. Let $q^{*}=\gamma\left(t_{0}\right)$ where $t_{0}=\sup \{t: r(\gamma(t))=\alpha\}$. Replacing $q$ by $q^{*}$ we then have that $r(\gamma(t))>\alpha$ for all $t$ such that $0<t \leq \delta$ and some small $\delta \leq \frac{1}{2}$. Now replacing $p$ by $\gamma(t)$ for some $t$ close to $t_{0}$ if necessary we can join $p$ to $q^{*}$ by a unit speed geodesic $\bar{\gamma}$ in $L$ (with respect to the induced metric on $L)$ with $\bar{\gamma}(0)=q^{*}$. If we let $\bar{t}_{0}=\sup \{t: r(\bar{\gamma}(t))=\alpha\}$ and $q^{* *}=\gamma\left(\bar{t}_{0}\right)$ then $r(\bar{\gamma}(t))>\alpha$ for $t>\bar{t}_{0}$ and $r\left(q^{* *}\right)=\alpha$. Thus, we see that, after making the above replacements if necessary and a reparameterization of $\gamma$, we may assume that $p$ is connected to $q$ by a unit speed geodesic in $L$ with respect to the induced metric on $L$ and with $\gamma(0)=q, \gamma(\delta)=p$ and $r(\gamma(t))>\alpha$ for $0<t \leq \delta \leq \frac{1}{2}$.

Now the hypothesis $S_{\partial_{r}}^{N_{\alpha}} \leq 0$ means that if we choose coordinates $x^{1}, \ldots, x^{n-1}$ on $N_{\alpha}$ and set $x^{n}=r-\alpha$ then $x^{1}, \ldots, x^{n}$ give a coordinate system on $\mathscr{U}$ such that Hess $x^{n} \leq 0$, when $x^{n}=0$. Let $\varphi: \mathscr{U} \rightarrow V \subset \mathbb{R}^{n}$ denote this coordinate map. Now on a possibly smaller neighborhood $\mathscr{U}^{\prime}$ of $q$ we may express $L$ as the graph of a function $f\left(x^{1}, \ldots, x^{n-1}\right)$, i.e. as the zero set of $g=f-x^{n}$ in $M$. Let $f(t)=f\left(x^{1} \circ \gamma(t), \ldots, x^{n-1} \circ \gamma(t)\right)$. Then, since image $(\gamma) \subset L$, 
we have $f(t)=x^{n}(\gamma(t))$. We have

$$
\begin{aligned}
f^{\prime \prime}(t) & =\text { Hess } f\left(\frac{\partial}{\partial t}, \frac{\partial}{\partial t}\right)+\left(\nabla_{\frac{\partial}{\partial t}} \frac{\partial}{\partial t}\right) f \\
& =\text { Hess } g\left(\frac{\partial}{\partial t}, \frac{\partial}{\partial t}\right)+\nabla_{\frac{\partial}{\partial t}} \frac{\partial}{\partial t} f+\text { Hess } x^{n}\left(\frac{\partial}{\partial t}, \frac{\partial}{\partial t}\right) .
\end{aligned}
$$

However, since $\gamma$ is a geodesic in $L$,

$$
\begin{aligned}
\nabla_{\frac{\partial}{\partial t} \frac{\partial}{\partial t} f} & =\left\langle\nabla_{\frac{\partial}{\partial t}} \frac{\partial}{\partial t}, \frac{\nabla g}{|\nabla g|}\right\rangle d f(\nabla g /|\nabla g|) \\
\nabla_{\frac{\partial}{\partial t}} \frac{\partial}{\partial t} f & =\left\langle\nabla_{\frac{\partial}{\partial t}} \frac{\partial}{\partial t}, \frac{\nabla g}{|\nabla g|}\right\rangle \frac{\langle d g, d f\rangle}{|\nabla g|} \\
& =-\left\langle\frac{\partial}{\partial t}, \nabla_{\frac{\partial}{\partial t}}\left(\frac{\nabla g}{|\nabla g|}\right)\right\rangle \frac{\langle d g, d f\rangle}{|\nabla g|} \\
& =-\left\langle\frac{\partial}{\partial t}, \nabla_{\frac{\partial}{\partial t}} \nabla g\right\rangle \frac{\langle d g, d f\rangle}{|\nabla g|^{2}} . \\
& =- \text { Hess } g\left(\frac{\partial}{\partial t}, \frac{\partial}{\partial t}\right) \frac{\langle d g, d f\rangle}{|\nabla g|^{2}} .
\end{aligned}
$$

Thus, since $x^{n}=r-\alpha$, we have

$$
f^{\prime \prime}(t)-\text { Hess } r\left(\frac{\partial}{\partial t}, \frac{\partial}{\partial t}\right)=\left(1-\frac{\langle d g, d f\rangle}{|\nabla g|^{2}}\right) \text { Hess } g\left(\frac{\partial}{\partial t}, \frac{\partial}{\partial t}\right) \text {. }
$$

Let $\mathscr{W} \subset \subset \mathscr{U}^{\prime}$ be an open set with compact closure $\overline{\mathscr{W}} \subset \mathscr{U}^{\prime}$ such that $\gamma([0, \delta]) \subset \mathscr{W}$. Let $T^{1} \mathscr{W}$ be the unit tangent bundle over $\mathscr{W}$ and set $\widetilde{\mathscr{W}}=\varphi(\mathscr{W})$. Let $X$ be a smooth section of $T^{1} \mathscr{W}$ which extends $\frac{\partial}{\partial t}$ along $\gamma$. Define the function $H_{X}=$ Hess $r(X, X) \circ \varphi^{-1}$ on $\widetilde{\mathscr{W}}$. We have

$$
\begin{aligned}
H_{X}\left(u^{1}, \ldots, u^{n}\right)= & H_{X}\left(u^{1}, \ldots, u^{n-1}, 0\right) \\
& +\int_{0}^{u^{n}} \frac{\partial}{\partial s} H_{X}\left(u^{1}, \ldots, u^{n-1}, s\right) d s
\end{aligned}
$$

for $\left(u^{1}, \ldots, u^{n}\right) \in \widetilde{\mathscr{W}}$.

Thus,

$$
H_{X}\left(u^{1}, \ldots, u^{n}\right)-H_{X}\left(u^{1}, \ldots, u^{n-1}, 0\right) \leq C u^{n},
$$

where $C$ is independent of $\left(u^{1}, \ldots, u^{n}\right) \in \widetilde{\mathscr{W}}$. In fact,

$$
C=\sup _{\tilde{\mathscr{W}}}\left\{\frac{\partial}{\partial u^{n}} H_{X}\left(u^{1}, \ldots, u^{n}\right)\right\} .
$$


We have

$$
H_{X}\left(u^{1}, \ldots, u^{n-1}, 0\right)=\text { Hess } r(X, X) \leq 0 .
$$

Consequently, if $u^{1}=x^{1}(\gamma(t)), \ldots, u^{n}=x^{n}(\gamma(t))$ and $X=\frac{\partial}{\partial t}$ then

$$
\begin{aligned}
H_{X}\left(x^{1}(\gamma(t)), \ldots, x^{n}(\gamma(t))\right) & =\text { Hess } r\left(\frac{\partial}{\partial t}, \frac{\partial}{\partial t}\right) \\
& \leq C x^{n}(\gamma(t))=C f(t) .
\end{aligned}
$$

Thus we have

$$
f^{\prime \prime}(t)-C f(t) \leq\left(1-\frac{\langle d g, d f\rangle}{|\nabla g|^{2}}\right) \text { Hess } g\left(\frac{\partial}{\partial t}, \frac{\partial}{\partial t}\right) .
$$

Now by choosing $\delta$ smaller if necessary we can assume that

$$
\langle d g, d f\rangle /|\nabla g|^{2} \leq \frac{1}{2}
$$

since $\left.d f\right|_{\gamma(0)}=0$, because $L$ and $N_{\alpha}$ are tangent at $p$. Thus it remains to show that $f^{\prime \prime}(t)-C f(t)>0$ for some $t$ with

$$
0<t<\delta \leq \frac{1}{2}
$$

We may assume $C=1$ since we can replace $f(t)$ by $f\left(\sqrt{C^{-1}} t\right)$ if necessary. Now, with the aim of reaching a contradiction assume that

$$
f^{\prime \prime}(t) \leq f(t)
$$

for all $t, 0<t<\delta \leq \frac{1}{2}$. Note that $f(0)=f^{\prime}(0)=0$ since $L$ and $N_{\alpha}$ are tangent at $p$.

Let $t_{0} \in(0, \delta)$. By the mean value theorem there is a $t^{*} \in\left(0, t_{0}\right)$ so that

$$
f^{\prime}\left(t^{*}\right)=\frac{f\left(t_{0}\right)-f(0)}{t_{0}}=\frac{f\left(t_{0}\right)}{t_{0}}
$$

Note that $f\left(t_{0}\right)>0$ by our choice of $\gamma$ and $\delta$. By another application of the mean value theorem there is a $t_{1}$ with $0<t_{1}<t^{*}<t_{0}$ so that from above we have

$$
f^{\prime \prime}\left(t_{1}\right)=\frac{f^{\prime}\left(t^{*}\right)}{t^{*}}=\frac{f\left(t_{0}\right)}{t^{*} t_{0}} .
$$

Our assumption, and the fact that $t^{*}, t_{0}<\delta \leq \frac{1}{2}$, gives

$$
f\left(t_{1}\right) \geq f^{\prime \prime}\left(t_{1}\right) \geq 2 f\left(t_{0}\right) .
$$

Repeat this procedure with $t_{1}$ replacing $t_{0}$ to get a $t_{2}<t_{1}$ with

$$
f\left(t_{2}\right) \geq 4 f\left(t_{0}\right) \text {. }
$$


Continuing this procedure gives

$$
f\left(t_{k}\right) \geq 2^{k} f\left(t_{0}\right) \text {. }
$$

This is a contradiction since the continuity of $f$ on $[0, \delta]$ implies that $f$ is uniformly bounded. Thus we conclude that $f^{\prime \prime}\left(t^{\prime}\right)-C f\left(t^{\prime}\right)>0$ for some $t^{\prime} \in(0, \delta)$. This together with our previous computations implies that

$$
0<\text { Hess }\left.g\left(\frac{\partial}{\partial t}, \frac{\partial}{\partial t}\right)\right|_{t^{\prime}} .
$$

But

$$
\text { Hess }\left.g\left(\frac{\partial}{\partial t}, \frac{\partial}{\partial t}\right)\right|_{t^{\prime}}
$$

is just $|\nabla g|\left\langle S_{\nu}^{L} \omega, \omega\right\rangle$ where $\omega=\left.\gamma_{*} \frac{\partial}{\partial t}\right|_{\gamma\left(t^{\prime}\right)}$ and $\nu=\frac{\nabla g}{|\nabla g|}$, and Lemma 3.5 holds.

In the following we establish some notation and record some facts to be used later.

3.6. Using the notation of the beginning of the section where the comparison theory was described, consider the case in which $\bar{M}=$ $S^{n}\left(\frac{1}{\sqrt{b}}\right)$ and $\bar{N}=\partial B_{r}$, where $B_{r}$ is a proper geodesic ball of radius $r$. We use the inward normal $\bar{\nu}$. Then, for any $\xi(t)$ as above, and for $0 \leq s<r<\pi / \sqrt{b}$, we have

$$
\frac{\|\bar{Y}(s)\|}{\|\bar{U}(s)\|}=\frac{\sin \sqrt{b}(r-s)}{\sin \sqrt{b} r}
$$

and

$$
\frac{d}{d s} \log \|\bar{Y}(s)\|=-\sqrt{b} \cot (\sqrt{b}(r-s)) .
$$

3.7. Take $\bar{M}=\mathbb{H}_{-a}^{n}$ the hyperbolic space of constant curvature $-a$. Let $\mathbb{H}_{-a}^{n-1}$ denote a totally geodesic submanifold of $\mathbb{H}_{-a}^{n}$ and let $\bar{N}=P_{r}$ where

$$
P_{r}=\left\{\exp _{\perp}^{\mathbb{H}_{-a}^{n-1}} r \nu(p): p \in \mathbb{H}_{-a}^{n-1}\right\}
$$

Take $\bar{\nu}$ to point toward $\mathbb{H}_{-a}^{n-1}$ i.e. let

$$
\bar{\nu}_{q}=-\left.\frac{d}{d t}\right|_{t=r} \exp _{\perp}^{\mathbb{H}_{-a}^{n-1}} t \nu(p)
$$

where $q=\exp _{\perp}^{\mathbb{H}_{-a}^{n-1}} r \nu(p)$. By symmetry, it suffices to take $n=2$ and use coordinates $x, y$ with metric

$$
d s^{2}=d x^{2}+\cosh (\sqrt{a} x) d y^{2} \text {. }
$$


We can take $\mathbb{H}_{-a}^{n-1}=\{x=0\}$ and $P_{r}=\{x=r\}$. An easy computation in these coordinates gives for $0 \leq s<r$,

$$
\begin{aligned}
\frac{\|\bar{Y}(s)\|}{\|\bar{U}(s)\|} & =\frac{\cosh \sqrt{a}(r-s)}{\cosh \sqrt{a} r}, \\
\frac{d}{d s} \log \|\bar{Y}(s)\| & =-\sqrt{a} \tanh (\sqrt{a}(r-s)) .
\end{aligned}
$$

3.8. Now let $r, \tilde{\mathscr{U}}, \mathscr{U}, \nu, N, N_{\alpha} \neq \varnothing$ etc. be as in the paragraph before Lemma 3.5. Suppose that the ambient manifold $M$ has nonnegative sectional curvature. Then $S_{\nu}^{N} \leq 0$ implies $S_{\partial_{r}}^{N_{\alpha}} \leq 0$ (recall that $\alpha>0$ ). To prove this, we can use the comparison theory quoted above with the choices $\xi(t)=\nu \circ \gamma(t), \bar{M}=\mathbb{R}^{n-1} \times \mathbb{R}=\mathbb{R}^{n}$ and $\bar{N}=\mathbb{R}^{n-1} \times\{0\} \subset \mathbb{R}^{n}$. We use (3.2) and the fact that

$$
\left.\frac{d}{d s} \log \|Y(s)\|\right|_{s=\alpha}=\left\langle S_{\partial_{r}}^{N_{\alpha}}(v), v\right\rangle /\|v\|^{2},
$$

where $v$ can be made equal to any element of $T N_{\alpha}$ by an appropriate choice of $\gamma(t)$. We also use that in this case the right-hand side of (3.2) will be zero.

3.9. We make the following definitions

$$
\begin{aligned}
& F\left(\kappa_{1}, a\right) \equiv \begin{cases}\frac{\operatorname{arctanh}\left(\frac{\kappa_{1}}{\sqrt{a}}\right)}{\sqrt{a}} & \text { if } 0<\kappa_{1}<\sqrt{a}, \\
\infty & \text { if } \kappa_{1} \geq \sqrt{a} \geq 0,\end{cases} \\
& G\left(\kappa_{2}, b\right) \equiv \begin{cases}\frac{\operatorname{arccot}\left(\frac{\kappa_{2}}{\sqrt{b}}\right)}{\sqrt{b}} & \text { if } b>0, \kappa_{2}>0, \\
\frac{1}{\kappa_{2}} & \text { if } b=0, \kappa_{2}>0, \\
\frac{\pi}{2 \sqrt{b}} & \text { if } b>0, \kappa_{2}=0, \\
\infty & \text { if } b=\kappa_{2}=0 .\end{cases}
\end{aligned}
$$

Here our convention is that $0<\operatorname{arccot}\left(\kappa_{2} / \sqrt{b}\right)<\pi / 2$. Also, $G(.,$.$) :$ $\mathbb{R}^{+} \times \mathbb{R}^{+} \rightarrow \overline{\mathbb{R}}$ is continuous, where $\mathbb{R}^{+}$is the nonnegative real numbers and $\overline{\mathbb{R}}$ is the extended real numbers.

3.10. If $N$ is a compact manifold and $\tau: N \rightarrow N$ is an isometric involution without fixed points then $\tau$ can be extended to $\left[0, s_{0}\right] \times N$ by $\tau(s, x)=\left(s_{0}-s, \tau(x)\right)$. This action also has no fixed points and so the group of 2 elements generated by $\tau$ acts by isometries on $\left[0, s_{0}\right] \times N$. This action gives a quotient manifold with boundary which we denote by $N_{\tau, s_{0}}$. 
Theorems 3.11 and 3.22 below allow us to replace $\varrho_{1}$ in the spectral results of $\S 2$ by $\kappa_{1}$ and $\kappa_{2}$ in some cases. If the hypothesis of Theorem 3.22(ii) below holds $\varrho_{1}$ can be replaced by boundary curvature bounds $\kappa_{2}$ and $\lambda_{1}$. Note that although most results in $\S 2$ use both $\varrho_{1}$ and $\varrho_{2}$, the number $\varrho_{2}$ was bounded for weakly convex domains in [D-L].

THeOREM 3.11. Let $D$ be a boundary-convex domain in a Riemannian manifold $M$. Suppose that $\partial D$ is smooth and compact. Let $K$ be the sectional curvature of $M$.

(i) If for $\kappa_{1}, \kappa_{2}, a, b \geq 0$ we have

$$
0<\kappa_{1} \leq-S_{\nu_{\text {in }}}^{\partial D} \leq \kappa_{2}
$$

and

$$
-a \leq K \leq b
$$

then $(r, p) \mapsto \exp _{\perp}^{\partial D} r \nu_{\text {in }}(p)$ is a diffeomorphism on $\left(0, r_{*}\right) \times \partial D$ where

$$
r_{*}=\min \left\{F\left(\kappa_{1}, a\right), G\left(\kappa_{2}, b\right)\right\} .
$$

(ii) If $0 \leq-S_{\nu_{\mathrm{in}}}^{\partial D} \leq \kappa_{2}$ and $0 \leq K \leq b$ then one of the following holds:

(a) $(r, p) \mapsto \exp _{\perp}^{\partial D} r \nu_{\text {in }}(p)$ is a diffeomorphism on $\left(0, G\left(\kappa_{2}, b\right)\right) \times$ $\partial D$ or

(b) There is an isometric involution $\tau$ of $\partial D$ without fixed points such that $\bar{D}$ is isometric to $\partial D_{\tau, s_{0}}$ where $s_{0}<2 G\left(\kappa_{2}, b\right)$.

Proof of Theorem 3.11. We will need several lemmas during the proof the first of which is

LEMMA 3.12. The map $(r, p) \mapsto \exp _{\perp}^{\partial D} r \nu_{\text {in }}(p)$ is a local diffeomorphism on $\left(0, R_{0}\right) \times \partial D$ where $R_{0}=G\left(\kappa_{2}, b\right)$ and $0 \leq \kappa_{2}$.

First we assume $b>0$ and $\kappa_{2}>0$. Let $R_{0}=\frac{\operatorname{arccot}\left(\kappa_{2} / \sqrt{b}\right)}{\sqrt{b}}$. Invoke the comparison theory mentioned above with the choices $\bar{M}=$ $S^{n}\left(\frac{1}{\sqrt{b}}\right), \bar{N}=\partial B_{R_{0}}$ and $\bar{\nu}$ inward. Here $B_{R_{0}}$ is a geodesic ball of radius $R_{0}$ in $S^{n}\left(\frac{1}{\sqrt{b}}\right)$. We have

$$
S_{\bar{\nu}}^{\partial B_{R_{0}}}=-\sqrt{b} \cot \sqrt{b} R_{0}=-\kappa_{2} \leq S_{\nu_{\text {in }}}^{\partial D}
$$

and $\bar{K}=b$ so that (3.3) holds and gives

$$
\frac{\|Y(s)\|}{\|U(s)\|} \geq \frac{\|\bar{Y}(s)\|}{\|\bar{U}(s)\|}=\frac{\sin \sqrt{b}\left(R_{0}-s\right)}{\sin \sqrt{b} R_{0}}>0
$$


for $0<s<R_{0}$. Now since $\|Y(s)\|=\left\|d \exp _{\perp}^{\partial D} U(s)\right\|$ and $d \exp _{\perp}^{\partial D}$ is already nonsingular in the radial direction we conclude that $d \exp _{\perp}^{\partial D}$ is nonsingular at any $\xi \in v(\partial D)$ with $\xi=r \nu_{\text {in }}(x)$ as long as $0 \leq$ $r<R_{0}$. By the inverse function theorem, $\exp _{\perp}^{\partial D}$ is a diffeomorphism on some neighborhood of any such point. The cases where $b=0$ or $\kappa_{2}=0$ follow by continuity of $G$.

We now show that (i) of the theorem holds. Using Lemma 3.12 above we will show next, as an intermediate result, that each segment $S_{x}$ given by the image of

$$
r \mapsto \exp _{\perp}^{\partial D} r \nu_{\text {in }}(x), \quad 0<r<r_{*},
$$

does not intersect $\partial D$. Note the strict inequalities for $r$ above. We start by noticing that the set $F=\left(\exp _{\perp}^{\partial D}\right)^{-1}(\partial D)$ is closed and contains the zero section of $\nu(\partial D)$ as a union of connected components. Let $F_{0}$ be this union and let $F_{1}$ be the part of $F-F_{0}$ contained in the $\nu_{\text {in }}$ component(s) of $\nu(\partial D)-F_{0}$. Now on $\nu(\partial D)$ we have a smooth function $\rho$ given by $\rho(\xi)=\|\xi\|$. Let $\left.\rho\right|_{F_{1}}$ take a minimum value at some $\xi_{1} \in F_{1} \subset v(\partial D)$. Now if $\rho\left(\xi_{1}\right) \equiv \alpha_{1} \geq r_{*}$ we have our intermediate result. We will show that the assumption $\alpha_{1}<r_{*}$ leads to a contradiction.

From Lemma 3.12 , there is a small ball $\widetilde{B}_{\xi_{1}}(\delta)=\widetilde{B}$ containing $\xi_{1}$ as its center so that $\left.\exp _{\perp}^{\partial D}\right|_{\widetilde{B}}$ is a diffeomorphism onto its image say $\mathscr{U} \subset M$. Under this diffeomorphism $\widetilde{B} \cap F_{1}$ maps to $\mathscr{U} \cap \partial D$. By taking $\widetilde{B}$ smaller if necessary we can guarantee that $\widetilde{B} \cap F_{0}=$ $\varnothing$. Now, since at $\xi_{1}$ the function $\rho$ restricted to $F_{1}$ achieves the minimum $\rho\left(\xi_{1}\right)=\alpha_{1}<r_{*}$, we must have that at $q=\exp _{\perp}^{\partial D} \xi_{1}$, $\left.\left.\rho \circ\left(\left.\exp _{\perp}^{\partial D}\right|_{\tilde{\mathscr{U}}}\right)^{-1}\right|_{\mathscr{U} \cap \partial D} \equiv r\right|_{\mathscr{U} \cap \partial D}$ achieves the minimum $\alpha_{1}$ and so $\mathscr{U} \cap \partial D$ is tangent with $N_{\alpha_{1}}$ (defined before where here $N=\partial D$ and $\widetilde{\mathscr{U}}=\widetilde{B})$ at $q$ and every point $p \in \mathscr{U} \cap \partial D$ has $r(p) \geq \alpha_{1}$. Let $\partial_{r}$ be the vector field associated with $r$ on $\mathscr{U}$. Then at $q$ we must have either

$$
\left.\partial_{r}\right|_{q}=\nu_{\text {in }}(q) \text { or }\left.\partial_{r}\right|_{q}=-\nu_{\text {in }}(q) .
$$

In the first case we see that for $t$ a little smaller than $\alpha_{1}$ the point $\exp _{\perp}^{\partial D} t \nu_{\text {in }}\left(p_{*}\right)$ is contained in $M-\bar{D}$ where $p_{*}=\pi\left(\xi_{1}\right) \in \partial D$. This means that $\exp _{\perp}^{\partial D} t \nu_{\text {in }}\left(p_{*}\right)$ must have crossed $\partial D$ for some $0<t<$ $\alpha_{1}$ contradicting the minimality of $\rho$ occurring at $\xi_{1}$. Thus, $\partial_{r}=$ $-\nu_{\text {in }}(q)$. We will use the comparison theory again to show that

$$
\left\langle S_{\partial_{r}}^{N_{\alpha_{1}}} X_{q}, X_{q}\right\rangle<0
$$


for $X_{q} \in T_{q} N_{\alpha_{1}}=T_{q}(\mathscr{U} \cap \partial D)$. This together with Lemma $3.5 \mathrm{~A}$ implies $\left\langle S_{\nu_{\text {in }}}^{\partial D} X_{q}, X_{q}\right\rangle>0$ contradicting our hypothesis that $S_{\nu_{\mathrm{in}}}^{\partial D} \leq 0$. This then implies the desired intermediate result.

Claim. (3.13) holds i.e.

$$
\frac{\left\langle S_{\partial_{r}}^{N_{\alpha_{1}}} v, v\right\rangle}{\|v\|^{2}}<0
$$

for any $v \in T_{q} N_{\alpha_{1}}$.

Proof of Claim. We can arrange that $\xi(t)$ (see the first paragraph of $\S 3)$ is chosen so that

We will need to show that

$$
\left.\frac{d}{d s} \log \|Y(s)\|\right|_{s=\alpha_{1}}=\frac{\left\langle S_{\partial_{r}}^{N_{\alpha_{1}}} v, v\right\rangle}{\|v\|^{2}} .
$$

$$
\left.\frac{d}{d s} \log \|Y(s)\|\right|_{s=\alpha_{1}}<0 .
$$

First assume $a>0$. Use the comparison theory with $\bar{M}=\mathbb{H}_{-a}^{n}, P_{r_{0}}$ and $\bar{\nu}$ as above (see 3.7), where we now let

$$
r_{0}=\frac{1}{\sqrt{a}} \operatorname{arctanh}\left(\frac{\kappa_{1}^{*}}{\sqrt{a}}\right)
$$

and $\kappa_{1}^{*}<\min \left\{\sqrt{a}, \kappa_{1}\right\}$.

We have that

$$
S_{\bar{\nu}}^{P_{r_{0}}}=-\sqrt{a} \tanh \sqrt{a} r_{0}=-\kappa_{1}^{*}>-\kappa_{1} \geq S_{\nu_{\mathrm{un}}}^{\partial D}
$$

and, since $\bar{K}=-a \leq K$, we have that (3.2) holds and gives

$$
\frac{d}{d s} \log \|Y(s)\| \leq-\sqrt{a} \tanh \sqrt{a}\left(r_{0}-s\right)<0
$$

for $0 \leq s<\min \left\{G\left(\kappa_{2}, b\right), r_{0}\right\}$. We have that $\min \left\{G\left(\kappa_{2}, b\right), r_{0}\right\} \rightarrow$ $r_{*}$ as $\kappa_{1}^{*} \rightarrow \min \left\{\sqrt{a}, \kappa_{1}\right\}$ and so we may choose $\kappa_{1}^{*}$ so that $\alpha_{1}<$ $\min \left\{G\left(\kappa_{2}, b\right), r_{0}\right\}$. The claim for $a>0$ now follows by letting $\kappa_{1}^{*} \rightarrow \min \left\{\sqrt{a}, \kappa_{1}\right\}$. The case $a=0$ is proven similarly by using the comparison theory with $\mathbb{R}^{n}$ instead of $\mathbb{H}_{-a}^{n}$ and a sphere of radius $1 / \kappa_{1}$ instead of $P_{r_{0}}$.

We now have the intermediate result that the segments $S_{x}$ described above are contained in $D$. This implies that $L_{r}$ given by the image of $\partial D$ under

$$
x \mapsto \exp _{\perp}^{\partial D} r \nu_{\text {in }}(x)
$$

for $0<r<r_{*}$ is contained in $D$ (and does not intersect $\left.\partial D\right)$. 
Now we are in a position to show that $(r, p) \rightarrow \exp _{\perp}^{\partial D} r \nu_{\text {in }}(P)$ is one to one on $\left(0, r_{*}\right) \times \partial D$. This fails if there exists a point $p$, and $r_{2}>r_{1}$ with

$$
\exp _{\perp}^{\partial D} r_{1} \nu_{\text {in }}(p)=\exp _{\perp}^{\partial D} r_{2} \nu_{\text {in }}(p)
$$

or points $p_{1} \neq p_{2}$ with

$$
\exp _{\perp}^{\partial D} r_{1} \nu_{\text {in }}\left(p_{1}\right)=\exp _{\perp}^{\partial D} r_{2} \nu_{\text {in }}\left(p_{2}\right) .
$$

We can reduce to the case where $r_{1}<r_{2}$. To see this consider the case where $p_{1} \neq p_{2}$ and $r_{1}=r_{2}$. In case the two geodesic segments connecting $p_{1}$ and $p_{2}$ to $p_{3} \equiv \exp _{\perp}^{\partial D} r_{1} \nu_{\text {in }}\left(p_{1}\right)=\exp _{\perp}^{\partial D} r_{2} \nu_{\text {in }}\left(p_{2}\right)$ do not meet tangentially or antitangentially then we may extend the segment $\overline{p_{2} p_{3}}$ to a slightly longer geodesic segment say $\overline{p_{2} p_{4}}$ of length $r_{2}+\varepsilon$. Because $\overline{p_{1} p_{3}}$ and $\overline{p_{3} p_{4}}$ meet with an angle other than 0 or $\pi$ the usual corner cutting argument shows that $r_{5} \equiv \operatorname{dist}\left(p_{4}, \partial D\right)<r_{1}+\varepsilon$. Thus, if we choose a point $p_{5}$ in $\partial D$ a distance $r_{5}$ from $p_{4}$ then we can consider the segments $\overline{p_{2} p_{4}}$ and $\overline{p_{5} p_{4}}$ as our replacements. In other words, since $r_{5}<r_{2}+\varepsilon$ we just replace $p_{1}$ by $p_{5}, r_{2}$ by $r_{2}+\varepsilon$, and $r_{1}$ by $r_{5}$.

In case $\overline{p_{1} p_{3}}$ and $\overline{p_{2} p_{3}}$ meet antitangentially the reduction is trivial, we just extend $\overline{p_{1} p_{3}}$ slightly. If $\overline{p_{1} p_{3}}$ and $\overline{p_{2} p_{3}}$ meet tangentially then $r_{1}=r_{2}$ forces $p_{1}=p_{2}$ by uniqueness of geodesics with equal tangents.

Thus, all we need to do is show that assuming

$$
\exp _{\perp} r_{1} \nu_{\text {in }}\left(p_{1}\right)=\exp _{\perp}^{\partial D} r_{2} \nu_{\text {in }}\left(p_{2}\right), \quad r_{1}<r_{2}<r_{*},
$$

leads to a contradiction. We proceed with this assumption.

Consider the set $\widetilde{L}_{r_{2}}$ in $v(\partial D)$ given by

$$
\widetilde{L}_{r_{2}}=\left\{\xi \in \nu(\partial D): \xi=r_{2} \nu_{\text {in }}(x), x \in \partial D\right\} .
$$

Define $L_{r_{2}}$ and $\widetilde{\widetilde{L}}_{r_{2}}$ by $L_{r_{2}}=\exp _{\perp}^{\partial D}\left(\widetilde{L}_{r_{2}}\right)$ and

$$
\widetilde{\widetilde{L}}_{r_{2}}=\left(\exp _{\perp}^{\partial D}\right)^{-1} L_{r_{2}} \cap\left\{r \nu_{\mathrm{in}}(p): r \geq 0, p \in \partial D\right\} .
$$

Since $\partial D$ is compact and $\widetilde{\widetilde{L}}_{r_{2}}$ is closed without intersecting the zero section $F_{0}$, we see that $\left.\rho\right|_{\widetilde{L}_{r_{2}}}$ must achieve a minimum $\alpha_{2}$ at some $\xi \in$ $\widetilde{\widetilde{L}}_{r_{2}}$ which must have $\alpha_{2}=\rho(\xi) \leq r_{1}$ since (3.14) holds. Let $\tilde{\mathscr{U}}$ be a small neighborhood of $\xi$ such that $\left.\exp _{\perp}^{\partial D}\right|_{\tilde{\mathscr{U}}}$ is a diffeomorphism onto $\mathscr{U} \subset D$. Then $r=\rho \circ \exp _{\perp}^{\partial D}||_{\tilde{\mathscr{U}}}^{-1}$ constrained to $S_{r_{2}}=\exp _{\perp}^{\partial D}\left(\tilde{\mathscr{U}}_{\tilde{L}} \cap \widetilde{\widetilde{L}}_{r_{2}}\right)$ 
achieves a minimum at the point $q=\exp _{\perp} \xi$. Now, by our definition of $\widetilde{\widetilde{L}}_{r_{2}}$ and $S_{r_{2}}$ there must be a $\xi^{*} \in \widetilde{L}_{r_{2}}$ with $q=\exp _{\perp} \xi^{*}$ and for some small neighborhood $\widetilde{V}$ of $\xi^{*}$ the restricted map $\left.\exp _{\perp}^{\partial D}\right|_{\widetilde{V}}$ is a diffeomorphism onto an open set $V$. Define a function $r^{\prime}$ on $V$ by $r^{\prime}=\left.\rho \circ \exp _{\perp}^{\partial D}\right|_{\widetilde{V}} ^{-1}$. Notice that $q \in V$ and $r^{\prime}(q)=r_{2}$. Thus, we have an $r_{2}$ level set for the function $r^{\prime}$ in $V$ which we denote by $N_{r_{2}}^{\prime}$. We have that $q \in \exp _{\perp}^{\partial D}\left(\widetilde{V} \cap \widetilde{L}_{r_{2}}\right)=N_{r_{2}}^{\prime} \subset S_{r_{2}}$. Hence, $\left.r\right|_{N_{r_{2}}^{\prime}}$ achieves a minimum $\alpha_{2}$ at $q \in N_{r_{2}}^{\prime}$ and so $N_{r_{2}}^{\prime}$ must lie in the part of $\mathscr{U}$ corresponding to values of $r$ greater than or equal to $\alpha_{2} \leq r_{1}$. Thus, we once again have two submanifolds tangent at a point $q$. Namely, the $r$ level set $N_{\alpha_{2}}$ and the $r^{\prime}$ level set $N_{r_{2}}^{\prime}$. The submanifold $N_{r_{2}}^{\prime}$ lies on the side of $N_{\alpha_{2}}$ corresponding to $r \geq \alpha_{2} . N_{\alpha_{2}}$ has an associated normal $\partial_{r}$ and $N_{r_{2}}^{\prime}$ has normal field $\partial_{r^{\prime}}=\operatorname{grad} r^{\prime}$. Now, if $\partial_{r^{\prime}}=\partial_{r}$ at $q$ then the two geodesics

$$
t \mapsto \exp _{\perp} t \nu_{\text {in }}\left(\pi\left(\xi^{*}\right)\right), \quad 0<t \leq r_{2}
$$

and

$$
t \mapsto \exp _{\perp} t \nu_{\text {in }}(\pi(\xi)), \quad 0<t \leq \alpha_{2},
$$

are tangent at $q=\exp _{\perp} \alpha_{2} \nu_{\text {in }}(\pi(\xi))=\exp _{\perp} r_{2} \nu_{\text {in }}\left(\xi^{*}\right)$. But, by uniqueness of geodesics with equal tangents and the fact that $r_{2}>r_{1} \geq \alpha_{2}$, the geodesic $t \mapsto \exp _{\perp} t \nu_{\text {in }}\left(\pi\left(\xi^{*}\right)\right), 0 \leq t \leq r_{2}$, must hit $\partial D$ at $\pi(\xi)$ for $t$ with $0<t \leq r_{2}$, a contradiction of the intermediate result obtained in the first part of the proof since $r_{2}<r_{*}$. Now, the case $\partial_{r^{\prime}}=-\partial_{r}$ is impossible since by (3.13) and Lemma 3.5A (choose $\nu=\partial_{r^{\prime}}$ and $L=N_{r_{2}}^{\prime}$ ) we would have $S_{\partial_{r^{\prime}}}^{N_{r_{2}}^{\prime}}>0$. But (3.13) applies to $N_{r_{2}}^{\prime}$ also so that $S_{\partial_{r^{\prime}}}^{N_{r_{2}}^{\prime}}<0$, a contradiction. Thus (3.14) cannot hold and this completes the proof of (i).

The following elementary lemma plays a crucial role in the proof of Theorem 3.11(ii).

LEMMA 3.15. Let $(\partial D)_{0}$ be a connected component of $\partial D$ and let $\widetilde{D}_{\alpha}$ be the band in $\nu(\partial D)$ given by

$$
\widetilde{D}_{\alpha}=\left\{r \nu_{\text {in }}(p): p \in(\partial D)_{0}, 0 \leq r \leq \alpha\right\} .
$$

Suppose that $\exp _{\perp}^{\partial D}$ is a local diffeomorphism on $\widetilde{D}_{\alpha}$. If $\exp _{\perp}^{\partial D}$ maps $\partial \widetilde{D}_{\alpha}$ into $\partial D$ and interior $\left(\widetilde{D}_{\alpha}\right)$ into interior $(D)$ then in fact 
$\exp _{\perp}^{\partial D}\left(\widetilde{D}_{\alpha}\right)=\bar{D}$. In particular $\partial D$ has at most 2 connected components.

Proof. Give $\bar{D}$ the relative topology inherited from $M$. We show that $\exp _{\perp}^{\partial D}\left(\widetilde{D}_{\alpha}\right)$ is open and closed in this topology. $\exp _{\perp}^{\partial D}\left(\widetilde{D}_{\alpha}\right)$ is closed since $\widetilde{D}_{\alpha}$ is compact and $\exp _{\perp}^{\partial D}$ is continuous. Let $x \in$ $\exp _{\perp}^{\partial D}\left(\widetilde{D}_{\alpha}\right)$. We show that $\exp _{\perp}^{\partial D} \widetilde{D}_{\alpha}$ contains an open neighborhood of $x$ in the $\bar{D}$ relative topology. If $x$ is interior to $D$ then for $p \in\left(\exp _{\perp}^{\partial D}\right)^{-1}(x)$ it must be that $p$ is interior to $\widetilde{D}_{\alpha}$ since otherwise our hypotheses guarantee that $x \in \partial D$. Thus we can find an open neighborhood $O \subset$ interior $\left(\widetilde{D}_{\alpha}\right)$ containing $p$ so that $\left.\exp _{\perp}^{\partial D}\right|_{o}$ is a diffeomorphism. Thus, since $\exp _{\perp}^{\partial D}(O) \subset$ interior $(D)$, we see that $\exp _{\perp}^{\partial D}(O)$ is a $\bar{D}$-open set containing $x$ and contained in $\exp _{\perp}^{\partial D}\left(\widetilde{D}_{\alpha}\right)$. Now, if $x \in \partial D$ then $p \in\left(\left.\exp _{\perp}^{\partial D}\right|_{\widetilde{D}_{\alpha}}\right)^{-1}(x)$ is in $\partial \widetilde{D}_{\alpha}$ and we can find an open set $O^{\prime}$ containing $p$ on which $\exp _{\perp}^{\partial D}$ is a diffeomorphism. Our hypotheses and continuity guarantee that for small $O^{\prime}$, $x \in \exp _{\perp}^{\partial D}\left(O^{\prime} \cap \widetilde{D}_{\alpha}\right)=\exp _{\perp}^{\partial D}\left(O^{\prime}\right) \cap \bar{D}$.

Proof of (ii). Once again we have that $\left.\rho\right|_{F_{1}}$ achieves a minimum. Call this minimum value $\alpha_{1}$ as before. The proof of (ii) is similar to but more difficult than that of (i) above. The program will be first to strive to prove the intermediate result that the geodesic segments $S_{x}$ given by

$$
r \mapsto \exp _{\perp}^{\partial D} r \nu_{\text {in }}(x), \quad 0<r<R_{0},
$$

do not intersect $\partial D$ for any $x \in \partial D$. Note that now the segments $S_{x}$ have length $R_{0} \equiv G\left(\kappa_{2}, b\right)$. Secondly we strive to show that

$$
(r, p) \mapsto \exp _{\perp}^{\partial D} r \nu_{\text {in }}(p)
$$

is one to one on $\left(0, R_{0}\right) \times \partial D$. That this map is a local diffeomorphism was proved in Lemma 3.12. We shall see that the only case in which we shall not be able to carry this program out is if the domain $D$ has the structure described in (iib) of the statement of the theorem.

Let $A$ be the set of points $\xi \in F_{1}$ such that $\rho(\xi)=\alpha_{1}$. We have two cases, namely $\pi(A) \neq \partial D$ and $\pi(A)=\partial D$.

Case I. $\pi(A) \neq \partial D$.

We shall prove that in this case (iia) holds. We first prove the intermediate result that the segments $S_{x}$ described above do not intersect $\partial D$. 
LEMMA 3.16. $\pi(A) \neq \partial D$ and $\alpha_{1}<R_{0}$ implies $\pi(A)$ has boundary points in $\partial D$, where $\partial D$ has the relative topology inherited from $M$.

The set $A$ is compact since it is closed and $\partial D$ is compact. By continuity $\pi(A)$ is compact and therefore closed in the Hausdorff space $\partial D$. We wish to show that in the case at hand $\pi(A)$ cannot also be open (in the induced topology on $\partial D$ ). To this end let us assume that $\pi(A)$ is open and seek a contradiction. We are assuming $\alpha_{1}<R_{0}$.

Since $\pi(A)$ is open and closed, we must have that $\pi(A)$ contains a connected component of $\partial D$ say $(\partial D)_{0}$. If $\partial D$ were connected so that $(\partial D)_{0}=\partial D$ we would be in Case II; $\partial D=\pi(A)$. However, even if $\partial D$ is not connected, we can use the connectedness of $D$ to show that $(\partial D)_{0} \subset \pi(A)$ implies $\pi(A)=\partial D$ (Case II). This is a contradiction since we are in Case I. We have $(\partial D)_{0} \subset \pi(A)$. The definition of $A$ implies that the set $\widetilde{A}_{\alpha_{1}}$ defined by

$$
\widetilde{A_{\alpha_{1}}}=\left\{\alpha_{1} \nu_{\text {in }}(p): p \in(\partial D)_{0} \subset \pi(A)\right\}
$$

is contained in $F_{1}$. Identify $\partial D$ with its image in $\nu(\partial D)$ under the zero section. With this notation, we have that $\widetilde{A}_{\alpha_{1}}$ is diffeomorphic to $(\partial D)_{0}$ and $\widetilde{A}_{\alpha_{1}} \cup(\partial D)_{0}$ forms the boundary of $\widetilde{D}_{\alpha_{1}}$, where

$$
\widetilde{D}_{\alpha_{1}}=\left\{r \nu_{\text {in }}(p): p \in(\partial D)_{0}, 0 \leq r \leq \alpha_{1}\right\} \text {. }
$$

Since $\alpha_{1}<R_{0}$, Lemmas 3.12 and 3.15 imply that

$$
\exp _{\perp}^{\partial D}\left(\widetilde{D}_{\alpha_{1}}\right)=\bar{D}
$$

Let $x \in \partial D$. The above considerations show that we can find $\xi \in$ $(\partial D)_{0} \cup \widetilde{A}_{\alpha_{1}}$ with $\exp _{\perp}^{\partial D} \xi=x$. If $\xi \in(\partial D)_{0}$ then since $(\partial D)_{0} \subset \pi(A)$ we have $x \in \pi(A)$. If $\xi \in{\widetilde{A_{\alpha}}}_{\alpha_{1}}$ then by the definition of ${\widetilde{A_{\alpha_{1}}}}_{\text {and }}$ ane established fact $\widetilde{A_{\alpha_{1}}} \subset F_{1}$ we see that there is a geodesic of length $\alpha_{1}$ connecting $\pi(\xi) \in \partial D$ to $\exp _{\perp}^{\partial D} \xi \in \partial D$, given by

$$
t \mapsto \exp _{\perp}^{\partial D} t \nu_{\text {in }}(\pi(\xi)) .
$$

By the Gauss lemma and the fact that $\widetilde{A}_{\alpha_{1}} \subset F_{1}$, we see that this geodesic meets $\partial D$ perpendicularly. Also, since $\alpha_{1}$ is a minimum of $\rho$ on $F_{1}$ and $\exp _{\perp}^{\partial D}$ preserves radial distance the above geodesic can only meet $\partial D$ at its endpoints. But this means that $x=\exp _{\perp}^{\partial D} \xi$ must also be in $\pi(A)$. In fact, the geodesic $t \mapsto \exp _{\perp}^{\partial D} t \nu_{\text {in }}(x), 0 \leq t \leq \alpha_{1}$, is just the reverse of the former geodesic and we see that $\alpha_{1} \nu_{\text {in }}(x) \in A$ 
and so $x \in \pi(A)$. Summarizing what we have done so far, we have shown that $\pi(A)$ open in $\partial D$ implies $\partial D=\pi(A)$ unless $\alpha_{1} \geq R_{0}$. Since we are in Case I, we conclude that in this case $\alpha_{1}<R_{0}$ is impossible unless $\pi(A)$ has boundary points in the relative topology on $\partial D$. This proves Lemma 3.16 .

Next we shall see even $\pi(A)$ having boundary points in $\partial D$ is, in Case I, incompatible with $\alpha_{1}<R_{0}$ so that in Case I, $\alpha_{1} \geq R_{0}$ and we will have our intermediate result. We continue to assume $\alpha_{1}<R_{0}$ and will once again reach a contradiction. Let $x_{0} \in \partial D$ be a boundary point of $\pi(A) \subset \partial D$. Let $\xi=\alpha_{1} \nu_{\text {in }}\left(x_{0}\right)$. Let $\widetilde{\mathscr{U}} \subset \nu(\partial D)$ be a connected open neighborhood of $\xi$ such that $\left.\exp _{\perp}^{\partial D}\right|_{\tilde{\mathscr{U}}}$ is a diffeomorphism onto a (connected) neighborhood $\mathscr{U}$ of $q=\exp _{\perp}^{\partial D} \xi$. We define $r=\rho \circ\left(\left.\exp _{\perp}^{\partial D}\right|_{\tilde{\mathscr{U}}}\right)^{-1}$ on $\mathscr{U}$. Now under this diffeomorphism $F_{1} \cap \tilde{\mathscr{U}}$ maps onto $\partial D \cap \mathscr{U}$ and $\left(\widetilde{L}_{\alpha_{1}} \cap \tilde{\mathscr{U}}, \xi\right)$ maps onto the pair $\left(N_{\alpha_{1}}, q\right)$ where $N_{\alpha_{1}} \subset L_{\alpha_{1}}$ is the level $r=\alpha_{1}$ submanifold as before. Since the minimum of $\left.r\right|_{\partial D \cap \mathscr{U}}$ occurs at $q$ (because $\xi \in A$ ) we must have

$$
T_{q} \partial D=T_{q} N_{\alpha_{1}}
$$

Also, just as in the proof of part (i) of the theorem $\partial_{r}=-\nu_{\text {in }}$ at $q$. Now $\pi$ is a submersion with $d \pi$ an isomorphism when restricted to $T_{\xi}\left(F_{1} \cap \widetilde{\mathscr{U}}\right)=T_{\xi}\left(\widetilde{L}_{\alpha_{1}} \cap \widetilde{\mathscr{U}}\right)$. It follows, after taking $\widetilde{\mathscr{U}}$ smaller if necessary, that $\left.\pi\right|_{F_{1} \cap \tilde{\mathscr{U}}}$ a diffeomorphism onto its image $\mathscr{W} \subset \partial D$ where $x_{0} \in \mathscr{W}$. Now since $x_{0}$ is a boundary point of $\pi(A)$, there must be a point $x_{0}^{\prime} \in \mathscr{W}$ with $x_{0}^{\prime} \notin \pi(A)$. This implies that $\xi^{\prime}=$ $\left.\pi^{-1}\right|_{F_{1} \cap \widetilde{\mathscr{U}}}\left(x_{0}^{\prime}\right)$ must satisfy $\rho\left(\xi^{\prime}\right)>\alpha_{1}$. Thus the point $p=\exp _{\perp}^{\partial D} \xi^{\prime} \in$ $\partial D \cap \mathscr{U}$ must satisfy $r(p)>\alpha_{1}$. Hence we are in a position to apply (3.8) and Lemma 3.5B to conclude that there exists $q_{1} \in \partial D \cap \mathscr{U}$ and $w \in T_{q_{1}} \partial D$ with $\left\langle-S_{\nu_{\text {in }}}^{\partial D} w, w\right\rangle<0$. This contradicts our hypothesis on the curvature of the boundary and so we are forced to conclude that, in Case I, we must have $\alpha_{1} \geq R_{0}=G\left(\kappa_{2}, b\right)$. This implies the intermediate result in this case.

Next we show that, in Case $\mathrm{I}$, the map $(r, p) \mapsto \exp _{\perp}^{\partial D} r \nu_{\text {in }}(p)$ is one to one on $\left(0, R_{0}\right) \times \partial D$. Arguing as in the proof of $(i)$, we see that we just need to show that assuming

$$
\exp _{\perp}^{\partial D} r_{1} \nu_{\text {in }}\left(p_{1}\right)=\exp _{\perp}^{\partial D} r_{2} \nu_{\text {in }}\left(p_{2}\right), \quad r_{1}<r_{2}<R_{0},
$$

leads to a contradiction. As before, we consider the sets $\widetilde{L}_{r_{2}}, L_{r_{2}}$, and $\widetilde{\widetilde{L}}_{r_{2}}$. With $\widetilde{\widetilde{L}}_{r_{2}}=\left(\exp _{\perp}^{\partial D}\right)^{-1} L_{r_{2}} \subset \nu(\partial D)$ as before we still have 
$\widetilde{\widetilde{L}}_{r_{2}} \cap F_{0}=\varnothing$ where $F_{0}$ is the zero section of $\nu(\partial D)$ (identified with $\partial D)$. Now $\left.\rho\right|_{\widetilde{L}_{r_{2}}}$ achieves a minimum which we again call $\alpha_{2}$. Let $A^{\prime}$ be the set of points $\xi \in \widetilde{\widetilde{L}}_{r_{2}}$ for which $\rho(\xi)=\alpha_{2}$. Now $\pi\left(A^{\prime}\right)$ is closed. If $\pi\left(A^{\prime}\right)$ is also open then $(\partial D)_{0} \subset \pi\left(A^{\prime}\right)$, where $(\partial D)_{0}$ is a connected component of $\partial D$. Assume then that this is the case. Then we see that the set

$$
\widetilde{A^{\prime}}=\left\{\alpha_{2} \nu_{\text {in }}(x): x \in(\partial D)_{0}\right\}
$$

is contained in $\widetilde{\widetilde{L}}_{r_{2}}$ and hence $\exp _{\perp}^{\partial D} \widetilde{A}^{\prime} \subset L_{r_{2}}$. Also, we have seen in the proof of (i) that we have, for all $x \in(\partial D)_{0}$,

$$
-\left.\frac{d}{d t} \exp _{\perp}^{\partial D} t \nu_{\text {in }}(x)\right|_{t=r_{1}}=\left.\frac{d}{d t} \exp _{\perp}^{\partial D} t \nu_{\text {in }}\left(\pi\left(\xi^{*}\right)\right)\right|_{t=r_{2}}
$$

where $\xi^{*} \in L_{r_{2}}$ and $\exp _{\perp}^{\partial D} \xi^{*}=q$ as before. Thus we have the following fact:

Fact 3.18. For any $x \in(\partial D)_{0}$ the geodesic

$$
\gamma: t \mapsto \exp _{\perp}^{\partial D} t \nu_{\text {in }}(x), \quad 0<t \leq \alpha_{2}+r_{2},
$$

connects $x \in \pi\left(A^{\prime}\right)$ to $\partial D$ and is perpendicular to $\partial D$ at its endpoints.

The above geodesic never intersects $\partial D$ for $0<t<\alpha_{2}+r_{2}$ by the intermediate result since $\alpha_{2}, r_{2}<R_{0}$. Thus it must be that $\alpha_{2}+$ $r_{2} \geq \alpha_{1}$. In particular $\alpha_{1}<2 R_{0}$. Let us agree to denote $\alpha_{2}+r_{2}$ by $\alpha_{3}$. Notice that since $(\partial D)_{0} \subset \pi\left(A^{\prime}\right)$ the number $\alpha_{3}=\alpha_{2}+r_{2}$ is independent of our choice of $x \in(\partial D)_{0}$, the starting point of the above geodesic. Thus $(r, p) \mapsto \exp _{\perp}^{\partial D} r \nu_{\text {in }}(p)$ maps the boundary (resp. interior) of $\left[0, \alpha_{3}\right] \times(\partial D)_{0}$ into the boundary (resp. interior) of $\bar{D}$. Now apply the following lemma, with $\alpha=\alpha_{3}$ and $U=(\partial D)_{0}$. Let

$$
\mathscr{L}(x)=\inf \left\{t: \exp _{\perp}^{\partial D} t \nu_{\text {in }}(x) \in \partial D\right\}
$$

LEMMA 3.19. If $U$ is an open subset of $\partial D$ contained in $\{x \in \partial D$ : $\mathscr{L}(x)=\alpha\}, \alpha<2 R_{0}$ and

$$
\left.\frac{d}{d t} \exp _{\perp}^{\partial D} t \nu_{\mathrm{in}}(x)\right|_{t=\alpha}
$$


is perpendicular to $\partial D$ for all $x \in U$ then $(r, p) \mapsto \exp _{\perp}^{\partial D} r \nu_{\text {in }}(p)$ is a local diffeomorphism on $[0, \alpha] \times U$.

Proof. It suffices to show that if $\xi=r \nu_{\text {in }}(p)$ for some $p \in U$ and $0 \leq r \leq \alpha$ then $\left.d \exp _{\perp}^{\partial D}\right|_{\xi}$ is a linear isomorphism. Since $d \exp _{\perp}^{\partial D}$ is always nonsingular in the radial direction we just need to show for any curve $\xi(t)$ in $\nu(\partial D),\|\xi(t)\|=$ constant $\leq \alpha, v=\left.\frac{d}{d t} \xi(t)\right|_{t=0} \neq 0$ and $\xi(0)=\xi$ inward that

$$
\left.d \exp _{\perp}^{\partial D}\right|_{\xi} v \neq 0
$$

Consider the curve $\theta(t)$ in $\partial D$ given by $\pi(\xi(t))$. Let $\tilde{\theta}(t)=$ $\exp _{\perp}^{\partial D} \alpha \nu_{\text {in }}(\theta(t))$. Notice that $\tilde{\theta}(t)$ is a curve in $\partial D$. Since the geodesic

$$
t \mapsto \exp _{\perp}^{\partial D} t \nu_{\text {in }}(x)
$$

where $0 \leq t \leq \alpha$, is normal to $\partial D$ at both end points, we can consider the reverse geodesic and we have

$$
\exp _{\perp}^{\partial D} s \nu_{\text {in }}(\theta(t))=\exp _{\perp}^{\partial D}(\alpha-s) \nu_{\text {in }}(\tilde{\theta}(t)) .
$$

From this we see that $\exp _{\perp}^{\partial D} \alpha \nu_{\text {in }}(\tilde{\theta}(t))=\theta(t)$. Hence, $\theta^{\prime}(0) \neq 0 \Rightarrow$ $\tilde{\theta}^{\prime}(0) \neq 0$. Let $Y(s)$ be the $\partial D$-Jacobi field given by

$$
Y(s)=\left.\frac{d}{d t} \exp _{\perp}^{\partial D} s \nu_{\text {in }}(\theta(t))\right|_{t=0}
$$

and let $\widetilde{Y}(s)$ be given by

$$
\tilde{Y}(s)=\left.\frac{d}{d t} \exp _{\perp}^{\partial D} s \nu_{\mathrm{in}}(\tilde{\theta}(t))\right|_{t=0} .
$$

Then from (3.20), we have $\tilde{Y}(\alpha-s)=Y(s)$. Now $\left.d \exp _{\perp}^{\partial D}\right|_{\xi} v=$ $Y\left(r_{3}\right)$ where $r_{3}=|\xi|$. If $r_{3} \leq \alpha / 2 \quad\left(<R_{0}\right)$ then by (3.12), $Y\left(r_{3}\right) \neq 0$. If $r_{3}>\alpha / 2$ then $\alpha-r_{3}<R_{0}$ and so

$$
Y\left(r_{3}\right)=\tilde{Y}\left(\alpha-r_{3}\right) \neq 0
$$

since

$$
\widetilde{Y}\left(\alpha-r_{3}\right)=\left.\frac{d}{d t} \exp _{\perp}^{\partial D}\left(\alpha-r_{3}\right) \nu_{\text {in }}(\tilde{\theta}(t))\right|_{t=0}
$$

and $\tilde{\theta}^{\prime}(0) \neq 0$. This proves the lemma.

In particular, we have, in the present case, that

$$
(r, p) \mapsto \exp _{\perp}^{\partial D} r \nu_{\text {in }}(p)
$$


is a local diffeomorphism from $\left[0, \alpha_{3}\right] \times(\partial D)_{0}$ into $\bar{D}$. From this and the comment just before Lemma 3.19, we see that Lemma 3.15 can be applied to conclude that

$$
\left\{\exp _{\perp}^{\partial D} r \nu_{\text {in }}(p):(r, p) \in\left[0, \alpha_{3}\right] \times(\partial D)_{0}\right\}=\bar{D}
$$

But this implies that in fact $\alpha_{3}=\alpha_{1}$. It follows as before that

$$
\pi(A)=\partial D
$$

which is Case II. Since we are in Case I, we are led to conclude that $\pi\left(A^{\prime}\right)$ is not open in $\partial D$ and thus contains a boundary point. Thus, arguing as in the paragraph before (3.17), once again we are in a position to use (3.8) and Lemma 3.5B to reach a contradiction. Thus $(r, p) \mapsto \exp _{\perp}^{\partial D} r \nu_{\text {in }}(p)$ is a diffeomorphism on $\left(0, R_{0}\right) \times \partial D$.

Case II. $\pi(A)=\partial D$.

LEMMA 3.21. In case $\pi(A)=\partial D$ if $\alpha_{1} \geq 2 R_{0}$ then $(r, p) \mapsto$ $\exp _{\perp}^{\partial D} r \nu_{\text {in }}(p)$ is a diffeomorphism on $\left[0, R_{0}\right) \times \partial D$.

Proof. We already have the local diffeomorphism property by 3.12 so it suffices to show that the map is one to one. We once again show that (3.17) leads to a contradiction if $r_{2}<R_{0}$. This time it is much easier. Let $L_{r_{2}}, \widetilde{L}_{r_{2}}$, and $\widetilde{\widetilde{L}}_{r_{2}}$ be defined as before. If $\rho \mid \widetilde{\widetilde{L}}_{r_{2}}$ achieves the minimum $\alpha_{2}$ at say $\xi$ then the geodesic $t \mapsto \exp _{\perp}^{\partial D} t \nu_{\text {in }}(x), 0<$ $t \leq \alpha_{2}+r_{2}$ with $x=\pi(\xi)$ meets $\partial D$ for the first time at $t=\alpha_{2}+r_{2}$. However $\alpha_{2}+r_{2}<2 R_{0} \leq \alpha_{1}$ a contradiction to the meaning of $\alpha_{1}$.

We must finally consider the case $\pi(A)=\partial D$ and $\alpha_{1}<2 R_{0}$. This is the only case where the conclusions of (iia) of the theorem have not been shown to hold. It is this situation in fact where (iib) holds.

Let $r_{3}=\frac{\alpha_{1}}{2}-\varepsilon$ and $r_{2}=\frac{\alpha_{1}}{2}+\varepsilon$ for some $\varepsilon$, where $0<\varepsilon<$ $\left(2 R_{0}-\alpha_{1}\right) / 2$. Then $r_{3}+r_{2}=\alpha_{1}$ and $r_{3}<r_{2}<R_{0}$. We may choose $\varepsilon$ small enough so that $r_{3}, r_{2}>0$. Let $\widetilde{\widetilde{L}}_{r_{2}}$ be as before and consider the minimum $\alpha_{2}$ of $\rho$ on $\widetilde{\widetilde{L}}_{r_{2}}$. Clearly, $\alpha_{2} \leq r_{2}<R_{0}$. The distance from $p=\exp _{\perp}^{\partial D} r_{2} \nu_{\text {in }}(x)$ to $\partial D$, for any $x \in \partial D$, is (by assumption). less than or equal to $r_{3}$. Thus we see, by considering a geodesic from $p$ to $\partial D$ whose length realizes this distance, that in fact $\alpha_{2} \leq r_{3}<r_{2}$. Let this minimum occur at some point $\xi \in \widetilde{\widetilde{L}}_{r_{2}}$. Then arguing in a manner similar to the paragraph of 3.14 , we see that the geodesic

$$
t \rightarrow \exp _{\perp}^{\partial D} t \nu_{\text {in }}(\pi(\xi)),
$$


$0<t \leq \alpha_{2}+r_{2}$, meets $\partial D$ for the first time when $t=\alpha_{2}+r_{2}$ and is normal to $\partial D$ there. Hence, $\alpha_{2}=r_{3}$ since we must have $\alpha_{2}+r_{2}=\alpha_{1}$. Let $x \in \partial D$. By assumption the geodesic,

$$
t \rightarrow \exp _{\perp}^{\partial D} t \nu_{\text {in }}(x)
$$

$0 \leq t \leq \alpha_{1}$, hits $\partial D$ only at its end points. Consider the point $p \in D$ given by $p=\exp _{\perp}^{\partial D} r_{2} \nu_{\text {in }}(x)$. Let $q \in \partial D$ be a closest point of $\partial D$ to $p$ and let $r=\operatorname{dist}(p, \partial D)$. Since $\alpha_{1}=r_{3}+r_{2}$ we have that $r \leq r_{3}$ so that in particular $q \neq x$. On the other hand since $\alpha_{2}=r_{3}$ we must have $r_{3} \leq r$ and thus $r=r_{3}$. But $r$ is the distance from $p$ to $\partial D$ and this implies that the geodesic segment $t \mapsto \exp _{\perp}^{\partial D} t \nu_{\text {in }}(x), r_{2} \leq$ $t \leq r_{3}+r_{2}$, hits $\partial D$ normally. This means that the geodesic segment $t \mapsto \exp _{\perp}^{\partial D} t \nu_{\text {in }}(x), 0 \leq t \leq \alpha_{1}=r_{3}+r_{2}$, hits $\partial D$ normally at both end points. Since $x$ was an arbitrary point in $\partial D$, we can apply Lemma 3.19 to get that $(r, p) \rightarrow \exp _{\perp}^{\partial D} r \nu_{\text {in }}(p)$ is a local diffeomorphism from $\left[0, \alpha_{1}\right] \times \partial D$ onto $\bar{D}$. Next we show that this map is a local isometry.

By the Gauss lemma, it suffices to show that for all $v \in T_{p} \partial D$, we have

$$
\|v\|=\|Y(r)\|
$$

where $Y(r)=\left.\frac{d}{d t} \exp _{\perp}^{\partial D} r \nu_{\text {in }}(\theta(t))\right|_{t=0}$ and $\theta^{\prime}(0)=v$. As in the proof of Lemma 3.18 we define $\tilde{\theta}$ and $\tilde{Y}$ by

$$
\tilde{\theta}(t)=\exp _{\perp}^{\partial D} \alpha_{1} \nu_{\text {in }}(\theta(t))
$$

and

$$
\tilde{Y}(s)=\left.\frac{d}{d t} \exp _{\perp}^{\partial D} s \nu_{\text {in }}(\tilde{\theta}(t))\right|_{t=0}
$$

Notice that since we are in Case II, $\tilde{\theta}(t) \in \partial D$. We can use the comparison theory with $\bar{M}=\mathbb{R}^{n}, \bar{N}=\mathbb{R}^{n-1} \subset \mathbb{R}^{n}$ to conclude, using (3.2), that

$$
\frac{2\left\langle Y^{\prime}(s), Y(s)\right\rangle}{\|Y(s)\|^{2}}=\frac{d}{d s} \log \|Y(s)\|^{2} \leq 0
$$

i.e.

$$
\frac{d}{d s}\langle Y(s), Y(s)\rangle=2\left\langle Y^{\prime}(s), Y(s)\right\rangle \leq 0
$$

However a similar statement is true for $\tilde{Y}$. We have $\tilde{Y}\left(\alpha_{1}-s\right)=Y(s)$ and

$$
\begin{aligned}
0 \geq \frac{d}{d s}\langle Y(s), Y(s)\rangle & =\frac{d}{d s}\left\langle\tilde{Y}\left(\alpha_{1}-s\right), \tilde{Y}\left(\alpha_{1}-s\right)\right\rangle \\
& =-\left.\frac{d}{d r}\langle\tilde{Y}(r), \tilde{Y}(r)\rangle\right|_{r=\alpha_{1}-s} \geq 0
\end{aligned}
$$


Thus $\frac{d}{d s}\|Y(s)\|^{2}=0$, for $0 \leq s \leq \alpha_{1}$. And $\|Y(s)\|=\|Y(0)\|=\|v\|$ for $0 \leq s \leq \alpha_{1}$. Thus $(r, p) \mapsto \exp _{\perp}^{\partial D} r \nu_{\text {in }}(p)$ is a local isometry on $\left[0, \alpha_{1}\right] \times \partial D$. Define a map $\tau: \partial D \rightarrow \partial D$ by $p \mapsto \exp _{\perp}^{\partial D} \alpha_{1} \nu_{\text {in }}(p)$. This is clearly a local isometry, even more, it is one to one and hence an isometry. In fact, it is clear that $\tau$ is an involution and hence one to one. We can extend $\tau$ to an isometry of $\left[0, \alpha_{1}\right] \times \partial D$ by $\tau(r, p)=\left(\alpha_{1}-r, \tau(p)\right)$. This action is free. $Q=\left[0, \alpha_{1}\right] \times \partial D /\langle\tau\rangle$ is the quotient manifold (with boundary). It is clear that the map $Q \mapsto \bar{D}$ induced by $(r, p) \mapsto \exp _{\perp}^{\partial D} r \nu_{\text {in }}(p)$ is a local isometry. We just need to show that $Q \rightarrow \bar{D}$ is $1-1$. Notice that $Q$ is compact and so $Q \rightarrow \bar{D}$ is a covering space with a finite number of sheets. Let this number be $k$. Thus $\operatorname{vol} Q=k \operatorname{vol} D$ as well as $\operatorname{vol} \partial Q=k \operatorname{vol} \partial D$. However, it is clear from our construction that $\partial Q$ is isometric to $\partial D$ and so $\operatorname{vol} \partial Q=\operatorname{vol} \partial D$. It follows that $k=1$. This is equivalent to $Q \rightarrow \bar{D}$ being one to one. This completes the proof of Theorem 3.11 .

Theorem 3.22. Let $D$ be a boundary-convex domain in a Riemannian manifold $M$. Assume that $\partial D$ is compact and smooth. Assume $0 \leq-S_{\nu_{1 \mathrm{n}}}^{\partial D} \leq \kappa_{2}$ and $0 \leq K \leq b$. We then have the following

(i) If one of (a), (b) or (c) below holds then $(r, p) \mapsto \exp _{\perp}^{\partial D} r \nu_{\text {in }}(p)$ is a diffeomorphism on $\left(0, G\left(\kappa_{2}, b\right)\right) \times \partial D$.

(a) $K>0$ on all 2-planes in $T_{q} D$, for some point $q \in D$.

(b) $0 \neq S_{\nu_{\mathrm{n}}}^{\partial D}$ at some point in $\partial D$.

(c) $M$ has no compact totally geodesic submanifolds.

(ii) If there is a point $p \in \partial D$ such that the ball of radius $R_{1}$ centered at $\exp _{\perp}^{\partial D} R_{1} \nu_{\text {in }}(p)$ is contained in $\bar{D}$ then

$$
(r, p) \mapsto \exp _{\perp}^{\partial D} r \nu_{\text {in }}(p)
$$

is a diffeomorphism on $\left(0, \min \left\{G\left(\kappa_{2}, b\right), R_{1}\right\}\right) \times \partial D$.

Notice that we may take $R_{1}$ to be any number less than the inradius of $D$. In case $D$ is boundary convex we have, by a standard result $[\mathbf{L}-\mathbf{Y}]$, that the in-radius is greater than $\frac{\pi}{2 \sqrt{\lambda_{1}(D)}}$ so in this case we may take $R_{1}$ to be this number. This fact is relevant if one applies these results to the inverse spectral project of $\S 2$.

Proof. The hypotheses of Theorem 3.11(ii) hold. Now (a), (b) of (c) exclude the possibility that $\bar{D}=\partial D_{\tau, s_{0}}$ and so the conclusions of Theorem 3.11(iia) hold. Thus (i) holds. For (ii) notice that even in case $\bar{D}=\partial D_{\tau, s_{0}}$ holds we must have $R_{1} \leq \frac{s_{0}}{2}$ and the result follows easily. 
Corollary 3.23. Let $p \in \partial D$ and let $p_{0}=\exp _{\perp}^{\partial D} s_{0} \nu_{\text {in }}(p)$. Let $B\left(p_{0}, s_{0}\right)$ denote the geodesic ball of radius $s_{0}$ and center $p_{0}$. The number $s_{0}$ need not be less than the injectivity radius of $M$. If the hypotheses of Theorem 3.11(i) hold then $B\left(p_{0}, s_{0}\right) \subset \bar{D}$ if $s_{0} \leq r_{*}$. If the hypotheses of Theorem 3.11(ii) hold, and $D \neq \partial D_{\tau, s}$ for any $s<2 G\left(\kappa_{2}, b\right)$ and any $\tau$, then $B\left(p_{0}, s_{0}\right) \subset \bar{D}$ with $s_{0}=G\left(\kappa_{2}, b\right)$.

\section{REFERENCES}

[B] M. van den Berg, $A$ uniform bound on trace $\left(e^{t \Delta}\right)$ for convex regions in $R^{n}$ with smooth boundaries, Comm. Math. Phys., 92 (1984), 525-530.

[Bl] W. Blaschke, Kreis und Kugel, Chelsea Publishing Company, 1949.

[C] S. Y. Cheng, Eigenvalue comparison theorems and its geometric applications, Math Z., 143 (1975), 289-297.

[D-L] H. Donnelly and J. Lee, Heat kernel remainders and inverse spectral theory, to appear in Illinois J. Math.

[D-Li] H. Donnelly and P. Li, Lower bounds for eigenvalues of Riemannian manifolds, Michigan Math. J., 29 (1982), 149-161.

[Ga] M. Gage, Upper bounds for the first eigenvalue of the Laplace-Beltrami operator, Indiana Univ. Math. J., 29 (1980), 897-912.

[G] A. Gray, Comparison theorems for the volumes of tubes as generalizations of the Weyl tubes formula, Topology, 21 (1982), 201-228.

[H-K] E. Heintz and H. Karcher, A general comparison theorem with applications to volume estimates for submanifolds, Ann. Sci. Ec. Norm. Sup., 11 (1978), 451-470.

[L] J. Lee, Hearing the volume of a drum in hyperbolic space, preprint.

[L-Y] P. Li and S. T. Yau, Estimates of eigenvalues of a compact Riemannian manifold, Proc. Sympos. Pure Math., vol. 36, Amer. Math. Soc., Providence, RI, 1980 , pp. 205-239.

[M-S] H. McKean and I. M. Singer, Curvature and eigenvalues of the Laplacian, J. Differential Geom., 1 (1967), 43-69.

[R-S] D. B. Ray and I. M. Singer, R-torsion and the Laplacian on Riemannian manifolds, Adv. Math., 7 (1971), 145-210.

[S] S. Selby, editor, Standard Mathematical Tables, Chemical Rubber Co., Cleveland, Ohio, 1968.

[T] A. Treibergs, Approximating the area of a convex domain from a finite part of the spectrum: lecture on a theorem of Li and Yau, preprint.

[W] F. Warner, Extension of the Rauch comparison theorem to submanifolds, Trans. Amer. Math. Soc., 122 (1966), 341-356.

Received January 16, 1990 and in revised form August 27, 1990.

Purdue University

WEST LAFAYETTE, IN 47907

AND

TeXas Tech University

LUBBOCK, TX 79409-1042 



\section{PACIFIC JOURNAL OF MATHEMATICS EDITORS}

\author{
V. S. VARADARAJAN \\ (Managing Editor) \\ -University of California \\ Los Angeles, CA 90024-1555-05 \\ Herbert Clemens \\ University of Utah \\ Salt Lake City, UT 84112 \\ THOMAS ENRIGHT \\ University of California, San Diego \\ La Jolla, CA 92093
}

R. FINN

Stanford University

Stanford, CA 94305

Hermann FlaschKa

University of Arizona

Tucson, AZ 85721

VAUGHAN F. R. JONES

University of California

Berkeley, CA 94720

SteVen KerCKhoff

Stanford University

Stanford, CA 94305
C. C. MOORE

University of California

Berkeley, CA 94720

Martin ScharlemanN

University of California

Santa Barbara, CA 93106

\section{HAROLD STARK}

University of California, San Diego

La Jolla, CA 92093

\section{ASSOCIATE EDITORS}

\begin{tabular}{|c|c|c|c|c|}
\hline R. Arens & $\begin{array}{l}\text { E. F. BECKENBACH } \\
(1906-1982)\end{array}$ & Neumann & $\begin{array}{l}\text { F. WolF } \\
(1904-1989)\end{array}$ & K. Yoshida \\
\hline \multicolumn{5}{|c|}{ SUPPORTING INSTITUTIONS } \\
\hline \multicolumn{2}{|c|}{ UNIVERSITY OF ARIZONA } & \multicolumn{3}{|c|}{ UNIVERSITY OF OREGON } \\
\hline \multirow{2}{*}{\multicolumn{2}{|c|}{$\begin{array}{l}\text { UNIVERSITY OF BRITISH COLUMBIA } \\
\text { CALIFORNIA INSTITUTE OF TECHNOLOGY }\end{array}$}} & \multicolumn{3}{|c|}{ UNIVERSITY OF SOUTHERN CALIFORNIA } \\
\hline & & \multicolumn{3}{|c|}{ STANFORD UNIVERSITY } \\
\hline CALIFORNIA INSTITUTE OF TECHNOLOGY & UNIVERSITY OF CALIFORNIA & \multicolumn{3}{|c|}{ UNIVERSITY OF HAWAII } \\
\hline \multicolumn{2}{|c|}{ MONTANA STATE UNIVERSITY } & \multicolumn{3}{|c|}{ UNIVERSITY OF TOKYO } \\
\hline \multicolumn{2}{|c|}{ UNIVERSITY OF NEVADA, RENO } & \multicolumn{3}{|c|}{ UNIVERSITY OF UTAH } \\
\hline \multicolumn{2}{|c|}{ NEW MEXICO STATE UNIVERSITY } & \multicolumn{3}{|c|}{ WASHINGTON STATE UNIVERSITY } \\
\hline OREGON STA & UNIVERSITY & UNIVERS & OF WASHING & \\
\hline
\end{tabular}




\section{Pacific Journal of Mathematics}

Vol. 150, No. $1 \quad$ September, 1991

Jan Aarts, Charles Lemuel Hagopian and Lex Gerard Oversteegen, The orientability of matchbox manifolds $\ldots \ldots \ldots \ldots \ldots \ldots \ldots \ldots \ldots \ldots \ldots \ldots$

Roger W. Barnard, Carl Hanson Fitzgerald and Sheng Gong, The growth and $1 / 4$-theorems for starlike mappings in $\mathbf{C}^{n} \ldots \ldots \ldots \ldots \ldots \ldots \ldots$

Allan Berele, Trace rings for verbally prime algebras $\ldots \ldots \ldots \ldots \ldots \ldots \ldots 23$

Quo-Shin Chi, Curvature characterization and classification of rank-one

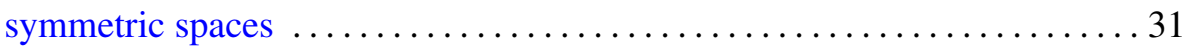

Harold Gerard Donnelly and Jeffrey Marc Lee, Domains in Riemannian

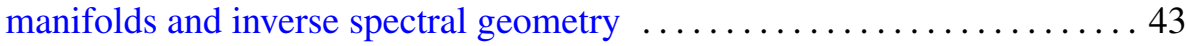

Clifford John Earle, Jr. and Patricia Lilaine Sipe, Families of Riemann

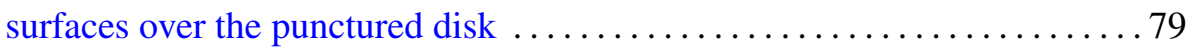

Mark P. Hughes, Dihedral group actions on homotopy complex projective

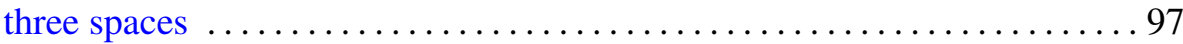

Robert F. Lax and Carl Widland, Gap sequences at a singularity ........111

Takahiko Nakazi, Bounded Hankel forms with weighted norms and lifting

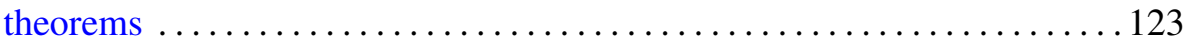

Douglas Murray Pickrell, Mackey analysis of infinite classical motion groups

Martha Rzedowski-Calderón and Gabriel Daniel Villa-Salvador,

Automorphisms of congruence function fields 167

Peter N-S Wong, Equivariant Nielsen fixed point theory for $G$-maps 